Sharif University of Technology
Scientia Iranica
Transactions C: Chemistry and Chemical Engineering
w
I RANw.scientiairanica.com

Research Note

\title{
Characterization of three-phase flow in porous media using the ensemble Kalman filter
}

\author{
S. Jahanbakhshi, M.R. Pishvaie* and R. Bozorgmehry Boozarjomehry \\ Department of Chemical and Petroleum Engineering, Sharif University of Technology, Tehran, P.O. Box 11365-11155, Iran.
}

Received 29 May 2016; received in revised form 14 September 2016; accepted 21 November 2016

\section{KEYWORDS}

Three-phase flow; Relative permeability; Capillary pressure; Ensemble Kalman filter;

Dimensionless sensitivity.

\begin{abstract}
In this study, the ensemble Kalman filter is used to characterize threephase flow in porous media through simultaneous estimation of three-phase relative permeabilities and capillary pressures from production data. Power-law models of relative permeability and capillary pressure curves are used and the associated unknown parameters are estimated by assimilating the measured historical data. The estimation procedure is demonstrated on a twin numerical setup with two different scenarios, in which a synthetic $2 \mathrm{D}$ reservoir under three-phase flow is considered. In the first scenario, all the endpoints are assumed to be known and only the shape factors are estimated during the assimilation process. In the second, all the endpoints and shape factors are estimated by assimilating observed data. Accurate estimation of the unknown model parameters is achieved by assimilating oil, water, and gas production rates of the producers and bottom-hole pressure of the injector. Moreover, sensitivity analysis of the observations with respect to the parameters defining the relative permeabilities and capillary pressures shows that for the most sensitive parameters, better estimation and lower uncertainty are obtained at the end of the assimilation process.
\end{abstract}

(C) 2017 Sharif University of Technology. All rights reserved.

\section{Introduction}

Several practical applications, such as enhanced oil recovery and solute transport in aquifers, involve simultaneous flow of two or three phases in the reservoir. Hence, it is a fundamental requirement to accurately model the multiphase flow and transport in the porous media [1]. Available models of multiphase flow are simply the extension of Darcy's law of single phase flow. Apparently, the only way to put the real physics of the flow into these conventional models is through capillary pressures and relative permeabilities. Thus, it

\footnotetext{
*. Corresponding author. Tel.: +98216616 6429;

Fax: +982166022853

E-mail addresses: samanjahanbakhshi@che.sharif.edu (S. Jahanbakhshi); pishvaie@sharif.edu (M.R. Pishvaie); brbozorg@sharif.edu (R. Bozorgmehry Boozarjomehry)
}

is of capital importance to correctly determine relative permeability and capillary pressure curves.

Reservoir simulation is a tool for decision-making processes involved in the development and management of petroleum reservoirs. A reservoir simulation model takes into account the impact of rock and fluid properties on the fluid flow through porous media. The model is then used to predict the future performance of the reservoir and assess sensitivity of these forecasts to uncertain reservoir parameters [2]. To improve the reliability of the model, it is beneficial to incorporate historical measurements in the simulation model, which is known as history matching in the petroleum literature [3].

Conventionally, two-phase relative permeabilities and capillary pressures are obtained from displacement experiments of core samples [4]. However, it is also possible to utilize production data to estimate relative 
permeabilities and capillary pressures through history matching [5,6]. Archer and Wong [7] were among the first investigators who determined the relative permeability curve by matching production data from a core displacement experiment. Many other researchers applied nonlinear optimization techniques to obtain an implicit estimation of relative permeabilities and/or capillary pressures from production data [8]. For example, Sigmund and McCaffery [9] applied nonlinear least-square procedure to determine relativepermeability curves for heterogeneous core samples taken from Alberta carbonate reservoirs. In their study, only shape factors of the relative permeability curves together with their standard errors were estimated by matching the observed recovery and pressure data. Kerig and Watson [10] extended the previous parameter estimation procedure by considering the use of several alternative functional forms, including the power-law and cubic spline models. They eventually concluded that cubic splines were more flexible than power-law models. These optimization algorithms require calculation of the Jacobian and/or Hessian matrix of the objective function (that is defined as the difference between the simulated results and the measurements). Henceforth, high computational cost and convergence to the local minima are two common shortcomings of these gradient-based methods [11]. Some investigators have also employed evolutionary algorithms, such as Genetic Algorithm (GA) and Simulated Annealing (SA) [12,13]. However, in these cases, there is no guarantee to find an optimal solution in a finite number of generations, and the convergence rate is relatively low. Recently, the widely used method in assisted history matching processes is the Ensemble Kalman Filter $($ EnKF), which is an efficient, recursive, and unbiased filter [14].

The EnKF is known as an attractive alternative for history matching problems because of its ease of implementation and computational efficiency for models with large number of variables. Also, EnKF is a sequential data assimilation scheme and is well suited for continuous reservoir model updating and closed-loop reservoir management problems [15]. Moreover, uncertainty assessment in reservoir characterization and performance predictions is becoming more important and, lately, more researchers have got involved in this area $[13,16]$. Since the EnKF can provide a measure of uncertainty along with history matching, it is also getting more attractive for the researchers working on uncertainty assessment. The EnKF is a sequential Monte Carlo method in which model states and covariance matrix are stored and propagated through an ensemble of model realizations. Compared to the other available methods, the EnKF requires no information about the gradient, which makes it more flexible to adapt to different types of model parameters and several commercial reservoir simulators [17]. The EnKF advantages as well as its limitations are thoroughly discussed in Evensen [18].

The EnKF has been applied to estimate various reservoir parameters, such as porosity and permeability fields [19], fluid contacts [20], and grid block transmissibility. Li and Yang [21] applied the EnKF to estimate multiple petro-physical parameters for the PUNQ-S3 model, which included estimation of vertical and horizontal permeability, porosity, and three-phase relative permeability curves. These curves were generated using power-law models. Unknown parameters of these models are of two types: shape factors and endpoints (which are discussed in Section 2.1). However, to simplify the model, only shape factors of these models were considered in the estimation process. They concluded that relative permeability could be estimated with good accuracy when the absolute permeability field was known. When relative permeability was tuned simultaneously along with porosity and absolute permeability, the accuracy of the estimated relative permeability was poor.

Li et al. [22] used a B-spline model for the twophase oil and water relative permeabilities. To ensure monotonicity of the relative permeability curves, a transformation was implemented to map the original parameters to the pseudo-parameters. These pseudoparameters were sequentially updated and adjusted toward their reference values, while the variance of the estimation decreased throughout the assimilation process using the EnKF. However, relative root-meansquare error of the parameters decreased more significantly before water breakthrough, in which the injected water reached the producing wells. Therefore, they concluded that the observation data collected prior to water breakthrough contributed more to the estimation of the relative permeability curve than those collected after water breakthrough. Seiler et al. [23] found that a significant improvement in the results of history matching was obtained by updating the relative permeabilities in addition to porosity and permeability fields and initial fluid contacts. Once more, power-law models of relative permeability curves were used and both the endpoints and the shape factors were estimated through history matching with the EnKF

Zhang et al. [24] used the EnKF to simultaneously estimate relative permeabilities and capillary pressures through matching the historical data, including water saturation profile, cumulative oil production, and pressure drop. Again, power-law model was used for both relative permeabilities and capillary pressures. They concluded, albeit qualitatively, that historical data were more sensitive to the relative permeabilities, 
especially water relative permeability, than capillary pressures. Their study, however, was limited to water flooding experiments in two-phase oil-water systems. Later, Zhang and Yang [25] used the EnKF to estimate three-phase relative permeabilities and capillary pressures for tight formations by assimilating historical data including oil, water, and gas production rates and cumulative oil production. Power-law models of relative permeabilities and capillary pressures were used and these prior models were updated during the assimilation process. Nevertheless, they considered the entry capillary pressures (i.e., endpoints of the powerlaw model) to be fixed to prevent poor estimation, and proposed to predetermine these values according to the prior knowledge. Moreover, the estimated shape factors of the capillary pressure curves were less accurate than the estimation of the relative permeability parameters, which was again qualitatively attributed to the less sensitivity of historical data to capillary pressure parameters.

This study is motivated by the work of $\mathrm{Li}$ et al. [26]. They considered a 2D, three-phase flow system and estimated endpoints and shape factors of the relative permeabilities by assisted history matching using the EnKF. They also found that distribution of the updated parameters tends to be Gaussian even if initial parameters are uniformly distributed. This is due to the fact that all the distributions are bound to be Gaussian in the EnKF. Furthermore, they proposed to integrate production data from multiple samples in the assimilation process to obtain more accurate estimation of multiphase flow parameters. The main contribution of this study is to advance the topic beyond the efforts of Li et al. [26] by including endpoints and shape factors of three-phase capillary pressure curves in the unknown parameter set. In other words, this study demonstrates joint estimation of three-phase relative permeability and capillary pressure curves by assimilating observation data. Furthermore, sensitivity of the observation data to the unknown model parameters is analyzed quantitatively, which clearly justifies the estimation results and the associated uncertainties of the unknown model parameters.

Here, an ensemble-based assimilation technique is developed and successfully applied in the simultaneous estimation of relative permeabilities and capillary pressures under three-phase flow condition in the porous media. Power-law models are used for both relative permeability and capillary pressure curves. Unknown model parameters of these models are adjusted progressively toward their reference values by assimilating observed historical data, including oil, water, and gas production rates of the producers and bottom-hole pressure of the injector. The estimation technique is validated in a synthetic five-spot 2D three-phase reservoir with two different scenar- ios. In the first, all the endpoints are assumed to be known and only the shape factors are estimated during the assimilation process. In the second, all the endpoints (including endpoints of the capillary pressure curves) and shape factors are estimated by assimilating observed data. Furthermore, the impacts of availability of the endpoints and ensemble size are evaluated, and quantitative sensitivity analysis of the observation data to the unknown parameters is performed.

\section{Methodology}

\subsection{Relative permeability and capillary pressure curves}

Direct measurement of three-phase relative permeabilities is difficult and time consuming; hence, empirical models are widely used [27]. A comprehensive description of the available correlations for the three-phase relative permeabilities along with their shortcomings is provided by Oliveira and Demond [28]. Based on the channel flow theory, the relative permeability of water and water-oil capillary pressure in a three-phase system are similar to those of the two-phase wateroil case [29]; and the relative permeability of gas and gas-oil capillary pressure are the same as those for the two-phase gas-oil case. The relative permeability of oil is then determined by interpolating two-phase data. Therefore, appropriate models of two-phase relative permeability and capillary pressure curves are initially needed.

Representative models of these curves are classified under two categories: parametric models and non-parametric models [26]. Non-parametric models, such as B-splines, have larger degrees of freedom, which make them more adaptable. However, if these models are used in the history matching process, a variable transformation is required to ensure that estimated relative permeabilities and capillary pressures are monotonic functions of saturation. Moreover, values estimated for a B-spline model frequently deviate from the reference values and, thus, the estimation suffers from non-uniqueness [11]. Parametric models, such as power-laws, are the most widely used models in multiphase flow studies.

Furthermore, the parameters defining a powerlaw relation carry more engineering information, and one may discuss their uncertainty in regard to time or sensitivity to the observation data. In this study, the empirical Corey-type (power-law) models are used to represent the two-phase relative permeability and capillary pressure curves. These models are simple and they also capture the trends of many experimental measurements.

For the oil-water system, relative permeability and capillary pressure functions are: 


$$
\left\{\begin{array}{l}
k_{r o}=a_{o}\left(1-S_{w D}\right)^{b_{o}} \\
k_{r w}=a_{w}\left(S_{w D}\right)^{b_{w}} \\
P_{c o w}=P_{c o w}^{*}\left(1-S_{w D}\right)^{n_{c o w}} \\
S_{w D}=\frac{S_{w}-S_{w c}}{1-S_{w c}-S_{o r w}}
\end{array}\right.
$$

where, $k_{r o}$ and $k_{r w}$ are relative permeabilities of oil and water, respectively; $S_{w}$ is the water saturation; $S_{w c}$ is the critical (or irreducible) water saturation; $S_{\text {orw }}$ is the residual oil saturation; and $a_{o}$ and $a_{w}$ are relative permeabilities of oil at $S_{w}=S_{w c}$ and water at $S_{w}=$ $1-S_{\text {orw }}$, respectively. Exponents $b_{o}$ and $b_{w}$ are shape factors of relative permeability curves; $P_{\text {cow }}^{*}$ is the oilwater capillary pressure at $S_{w}=S_{w c} ; n_{\text {cow }}$ is the shape factor of the capillary pressure curve; and $S_{w D}$ is the dimensionless water saturation.

For the gas-oil system, relative permeability and capillary pressure functions are:

$$
\left\{\begin{array}{l}
k_{r o g}=a_{o g}\left(1-S_{g D}\right)^{b_{o g}} \\
k_{r g}=a_{g}\left(S_{g D}\right)^{b_{g}} \\
P_{c g o}=P_{c g o}^{*}\left(S_{g D}\right)^{n_{c g o}} \\
S_{g D}=\frac{S_{g}-S_{g c}}{1-S_{w c}-S_{g c}-S_{o r g}}
\end{array}\right.
$$

where, $k_{r o g}$ and $k_{r g}$ are relative permeabilities of oil and gas, respectively; $S_{g}$ is the gas saturation; $S_{g c}$ is the critical gas saturation; $S_{\text {org }}$ is the residual oil saturation; and $a_{o g}$ and $a_{g}$ are relative permeabilities of oil at $S_{g}=S_{g c}$ and gas at $S_{g}=1-S_{w c}-S_{\text {org }}$, respectively. On the other hand, exponents $b_{o g}$ and $b_{g}$ are shape factors of relative permeability curves; $P_{c g o}^{*}$ is the gas-oil capillary pressure at $S_{g}=S_{g c}$; exponent $n_{c g o}$ is the shape factor of the capillary pressure curve; and $S_{g D}$ is the dimensionless gas saturation. Consequently, parameters of the relative permeabilities and capillary pressures are divided into two types: shape factors $\left(b_{o}, b_{w}, n_{\text {cow }}, b_{o g}, b_{g}\right.$, and $\left.n_{c g o}\right)$ and endpoints $\left(a_{o}, a_{w}, P_{c o w}^{*}, a_{o g}, a_{g}\right.$, and $\left.P_{c g o}^{*}\right)$. Essentially, $a_{o}=a_{o g}=1[11,26]$, because relative permeability is defined as the ratio of the effective permeability to the absolute oil permeability at the irreducible water saturation.

The oil relative permeability for the simultaneous flow of oil, water, and gas in the reservoir is calculated by Stone's model II method [30]. Stone proposed two empirical models of three-phase relative permeability that are the most popular in the petroleum industry [31]. These models are considered as the industry standard and have become a benchmark against which other models are compared. Stone's model II is a modified version of Stone's model I and better predicts oil relative permeability in three-phase flow systems, especially for water-wet reservoir rocks [32]. Moreover, it is not required to estimate the minimum residual oil saturation $\left(S_{o m}\right)$ in this model. Stone [30] further verified the validity of model II by comparing the residual oil saturation data predicted from this model with the experimental measurements given by Holmgren and Morse [33]. In addition, hysteresis effects can be easily taken into consideration by using the appropriate twophase data.

The form of Stone's model II used in this study is the modification proposed by Aziz and Settari [34], which is as follows [27]:

$$
k_{r o}=a_{o}\left[\left(\frac{k_{r o}}{a_{o}}+k_{r w}\right)\left(\frac{k_{r o g}}{a_{o g}}+k_{r g}\right)-\left(k_{r w}+k_{r g}\right)\right] \text {. }
$$

Stone's model II gives relative permeability of oil as a function of $S_{w}$ and $S_{g}$. Negative values may be obtained from this model, albeit they have no physical meaning. Thus, $k_{r o}$ is set to zero whenever the model predicts a negative value [28].

\subsection{State-space representation of system}

The discrete nonlinear state-space formulation of the system is represented as [35]:

$$
\begin{aligned}
& \mathbf{x}_{k}=\mathbf{M}_{k}\left(\mathbf{x}_{k-1}, \mathbf{u}_{k}\right)+\mathbf{w}_{k}, \\
& \mathbf{d}_{k}=\mathbf{H}_{k} \mathbf{x}_{k}+\mathbf{D}_{k},
\end{aligned}
$$

where $\mathbf{M}_{k}$ is the reservoir simulation model; $\mathbf{x}_{k}=$ $[\mathbf{s}, \mathbf{m}, \tilde{\mathbf{d}}]_{k}^{T}$ is the (augmented) state vector wherein $\mathbf{s}$ is $n_{s} \times 1$ system states (which consist of pressures, water and gas saturations, and solution gas/oil ratios for each grid block); $\mathbf{m}$ is $n_{m} \times 1$ model parameters that encounter constant dynamics (i.e., $\mathbf{m}_{k}=\mathbf{m}_{k-1}$ ); and $\tilde{\mathbf{d}}$ is $n_{d} \times 1$ predicted observations. $\mathbf{u}_{k}$ is the control inputs or boundary conditions and $\mathbf{w}_{k}$ is the dynamic system noise (or model error), which is a white Gaussian noise with covariance matrix $\mathbf{Q}_{k}$ of size $n_{d} \times n$ ( $n$ is the size of the state vector, $\left.n=n_{s}+n_{m}+n_{d}\right)$, i.e. $\mathbf{w}_{k} \sim \mathbf{N}\left(0, \mathbf{Q}_{k}\right)$. $\mathbf{d}_{k}$ is the vector of real observations of size $n_{d} \times 1$ and matrix $\mathbf{H}_{k}$ of size $n_{d} \times n$ is the operator mapping the state space to the observation space [18]:

$$
\mathbf{H}_{k} \equiv\left[\begin{array}{ll}
\mathbf{O} & \mathbf{I}
\end{array}\right]_{k}
$$

where $\mathbf{O}$ is the null matrix of size $n_{d} \times\left(n_{s}+n_{m}\right)$ and $\mathbf{I}$ is the identity matrix of size $n_{d} \times n_{d}$. Also, $\mathbf{D}_{k}=\left[\varepsilon_{1}, \varepsilon_{2}, \cdots, \varepsilon_{n_{d}}\right]_{k}$ is the vector of measurement perturbations of size $n_{d} \times 1$, which are sampled from a white Gaussian distribution with covariance matrix $\mathbf{R}_{k}$ of size $n_{d} \times n_{d}$, i.e. $\mathbf{D}_{k} \sim \mathbf{N}\left(0, \mathbf{R}_{k}\right)$.

As the state vector also includes the predicted observations, the relation between $\mathbf{d}_{k}$ and $\mathbf{x}_{k}$ can always be expressed by Eq. (5), regardless of the type of the relationship that exists between $\mathbf{d}_{k}$ and $\mathbf{s}_{k}$. In addition, because there is little theoretical knowledge of 
model error statistics, a rigorous procedure to properly account for modeling errors has not been established for multiphase flow in porous media [36]. Moreover, a common assumption in the parameter estimation applications is that the dominating errors in the model are due to the uncertainty in the parameters and, thus, dynamic model error is neglected $\left(\mathbf{Q}=\mathbf{O}_{n \times n}\right)$. Reservoir simulation model together with the initial and boundary conditions is described in Section 3.1.

\subsection{The ensemble Kalman filter}

The EnKF is a sequential Monte Carlo method in which a finite number of ensemble members are used to forecast error statistics and to approximate Kalman gain matrix for updating model variables [37]. The EnKF is the most suitable for high-dimensional discrete systems wherein storage and manipulation of the error covariance matrix are impossible or impractical [38]. In the EnKF, model states and system error covariance are stored and manipulated implicitly through an ensemble of model realizations. The EnKF is an efficient and easy-to-implement data assimilation method and has been successfully applied in history matching problems to estimate various parameters of the reservoir. Details of the EnKF theory and algorithms are described by Evensen [18]. The EnKF contains two consecutive steps; the first is the forecast step in which the reservoir simulator uses the updated model realizations from the previous time step to generate forecasted state vector for the current time where new observation data are available; in the second step, known as the assimilation step, the forecasted state vector is assimilated with new observations under a Bayesian framework.

For simplicity, time index of the variables is removed. The forecast ensemble perturbations or anomalies are obtained based on calculation of their difference from the mean of the ensemble. Then, the $i$ th vector of anomalies becomes $\mathbf{A}_{i}^{f}=\mathbf{x}_{i}^{f}-\mathbf{x}^{f}$ :

$$
\mathbf{A}^{f}=\left[\mathbf{x}_{1}^{f}-\mathbf{x}^{f}, \mathbf{x}_{2}^{f}-\mathbf{x}^{f}, \cdots, \mathbf{x}_{N}^{f}-\mathbf{x}^{f}\right],
$$

in which $\mathbf{x}_{i}^{f}$ is the $i$ th forecast vector, $N$ is the number of ensemble members, and $\mathbf{x}^{f}$ is the forecast of ensemble mean, as follows:

$$
\mathbf{x}^{f}=\frac{1}{N} \sum_{i=1}^{N} \mathbf{x}_{i}^{f} .
$$

The forecast of error covariance matrix is then calculated as:

$$
\begin{aligned}
\mathbf{P}^{f} & =\frac{1}{N-1} \sum_{i=1}^{N}\left(\mathbf{x}_{i}^{f}-\mathbf{x}^{f}\right)\left(\mathbf{x}_{i}^{f}-\mathbf{x}^{f}\right)^{T} \\
& =\frac{1}{N-1} \mathbf{A}^{f}\left(\mathbf{A}^{f}\right)^{T},
\end{aligned}
$$

wherein superscript $T$ denotes matrix transpose. In the assimilation step, the assimilated ensemble mean, ensemble perturbations, and ensemble members are obtained by employing Kalman filter equation:

$$
\begin{aligned}
& \mathbf{x}^{a}=\mathbf{x}^{f}+\mathbf{K}\left(\mathbf{d}-\mathbf{H} \mathbf{x}^{f}\right), \\
& \mathbf{A}^{a}=\mathbf{A}^{f}+\mathbf{K}\left(\mathbf{D}-\mathbf{H} \mathbf{A}^{f}\right), \\
& \mathbf{x}_{i}^{a}=\mathbf{A}_{i}^{a}+\mathbf{x}^{a} .
\end{aligned}
$$

Here, $\mathbf{K}$ is the Kalman gain matrix of size $n \times n_{d}$. Kalman gain is calculated based on the minimization of the analysis error covariance matrix [35]:

$$
\begin{aligned}
\mathbf{P}^{a} & =\frac{1}{N-1} \sum_{i=1}^{N}\left(\mathbf{x}_{i}^{a}-\mathbf{x}^{a}\right)\left(\mathbf{x}_{i}^{a}-\mathbf{x}^{a}\right)^{T} \\
& =\frac{1}{N-1} \mathbf{A}^{a}\left(\mathbf{A}^{a}\right)^{T}
\end{aligned}
$$

which results in [38]:

$$
\begin{aligned}
& \mathbf{K}=\mathbf{P}^{f} \mathbf{H}^{T}\left(\mathbf{H} \mathbf{P}^{f} \mathbf{H}^{T}+\mathbf{R}\right)^{-1}, \\
& \mathbf{P}^{a}=(\mathbf{I}-\mathbf{K H}) \mathbf{P}^{f} .
\end{aligned}
$$

At each assimilation stage, the system states, model parameters, and predicted observations are updated simultaneously.

Even though there is a great interest to use the EnKF for reservoir history matching, the method suffers from a couple of shortcomings. A basic assumption of the EnKF is that the updated state variables (primary reservoir-simulation variables) and updated static model parameters are statistically consistent. That is, at each assimilation step, updated ensemble of state variables is same as the ensemble of state variables obtained by running the reservoir simulator with updated ensemble of parameters from time zero. This consistency has been proved only for systems with Gaussian statistics in which there is a linear relation between model parameters and state variables [39-41]. However, the reservoir simulation equations are highly nonlinear and, consequently, this assumption can be invalid. A procedure to avoid inconsistency is to rerun the simulator with the latest updated ensemble of model parameters from time zero to the current time after each assimilation step. This procedure is referred to as half-iteration EnKF [20,40]. Obviously, the simulation re-run increases the computational cost. Thus, Wang et al. [20] proposed to do a re-run only if the relative change in the ensemble mean of the model parameters is larger than a threshold value [17]. However, in this study, we have considered a small synthetic reservoir model, which is described in Section 3.1. Subsequently, computational burden is not a critical challenge here and a simulation re-run is performed after each assimilation cycle. 


\subsection{Half-iteration ensemble-based assimilation procedure}

The implementation road map for estimation of relative permeability and capillary pressure curves is summarized below:

- Step 1: Representative models of relative permeabilities and capillary pressures are selected and initialized. A number of $N$ realizations from the prior knowledge of the unknown parameters are generated;

- Step 2: All the ensemble parameter sets are propagated with the reservoir simulator to generate the predicted observations for the next measurement time. Therefore, the state vector components for each ensemble member become:

$$
\begin{aligned}
& \mathbf{s}=\left[P_{o}, S_{w}, S_{g}, R_{s}\right]^{T} \quad \text { for each grid block, (16) } \\
& \mathbf{m}=\left[b_{o}, a_{w}, b_{w}, P_{\text {cow }}^{*}, n_{\text {cow }}, b_{o g}, a_{g}, b_{g}, P_{c g o}^{*}, n_{c g o}\right]^{T},
\end{aligned}
$$

$$
\begin{aligned}
\tilde{\mathbf{d}}= & {\left[\mathrm{WOPR}_{i}, \mathrm{WWPR}_{i}, \mathrm{WGPR}_{i}, \mathrm{WBHP}_{j}\right]^{T}, } \\
& \left\{\begin{array}{l}
i \in\{\text { Production wells }\} \\
j \in\{\text { Injection well }\}
\end{array}\right.
\end{aligned}
$$

- Step 3: When new observations become available, the EnKF algorithm is used to assimilate the real observations with the predicted ones, meanwhile relative permeability and capillary pressure parameters are updated;

- Step 4: The reservoir simulator is re-run with the updated ensemble of model parameters from time zero to the current time. Consequently, updated ensemble of system states, s, and predicted observations, $\tilde{\mathbf{d}}$, which is consistent with the updated ensemble of model parameters, is obtained;

- Step 5: The assimilation procedure is repeated until no more observations are available.

To evaluate the filter performance, Relative RootMean-Square Error (RRMSE) is calculated, which illustrates the dimensionless difference between the average estimated values and reference (or true) values, as:

$$
\operatorname{RRMSE}=\sqrt{\frac{1}{N_{p}} \sum_{i=1}^{N_{p}}\left[\frac{x_{i}^{\mathrm{avg}}-x_{i}^{\mathrm{true}}}{x_{i}^{\mathrm{true}}}\right]^{2}}
$$

wherein $x_{i}^{\text {avg }}$ and $x_{i}^{\text {true }}$ are the average and true values of the $i$ th model parameter, respectively, and $N_{p}$ is the total number of to-be-estimated parameters. As discussed previously, a twin numerical setup has been implemented for assessment purposes. Consequently, true values of to-be-estimated parameters are known (which will be discussed in Section 3.2) in order to examine the estimation performance. If the assimilation converges, RRMSE will decrease along the simulation time interval. However, RRMSE is just a traditional tool that has been employed in many other studies and does not guarantee the success of assimilation $[5,42]$.

\section{Case study}

\subsection{Reservoir model description}

A 2D synthetic reservoir is considered to simultaneously estimate relative permeabilities and capillary pressures under three-phase flow condition. The synthetic model is built in the commercial finitedifference-based simulator, ECLIPSE. ECLIPSE is a well-established and industry-reference simulator that has been widely applied as the forward model in the assisted history matching processes $[12,15]$. Accordingly, in this study, ECLIPSE software with a fully-implicit scheme is considered as the forward simulation model.

The heterogeneous reservoir model is divided into a uniform grid system of size $21 \times 21 \times 1$ with dimensions of $100 \times 100 \times 50 \mathrm{ft}$. No-flow boundary condition is assumed at all sides of the reservoir model, and the phases present in the reservoir are oil, water, and gas. Absolute permeability map is generated using sgsim module of SGeMS software [43] with isotropic Gaussian covariance in which major and minor ranges are set to 5 grid blocks. Figure 1 depicts the absolute log-permeability map, whose mean and variance are equal to 6 and $1 \mathrm{mD}$, respectively, and the well patterns. Porosity of the formation is $10 \%$. Absolute permeability and porosity are known and fixed during the assimilation process.

System states consist of (oil) pressure, water and gas saturations, and solution gas/oil ratio for each grid

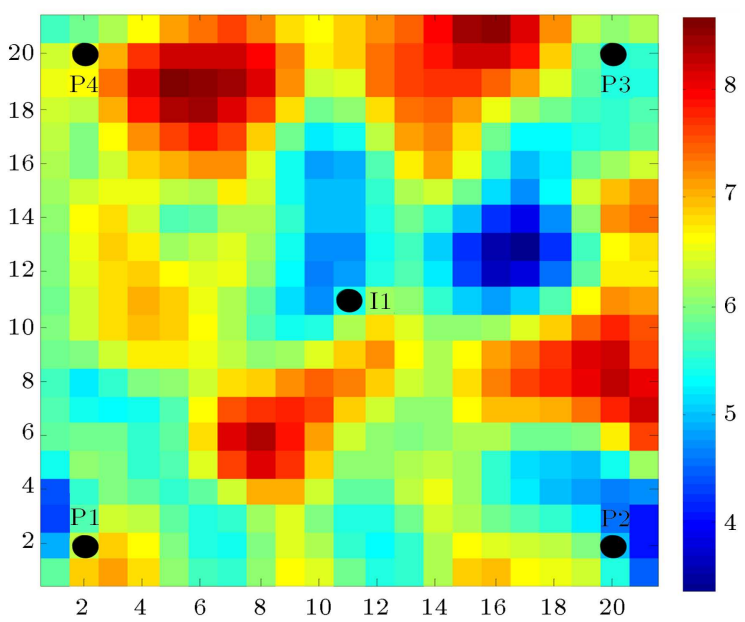

Figure 1. Absolute log-permeability field $(\mathrm{mD})$ and well locations of the synthetic $2 \mathrm{D}$ reservoir. 
Table 1. Well patterns and specifications.

\begin{tabular}{cccccc}
\hline I1 & P4 & P3 & P2 & P1 & Well \\
\hline Well type & Producer & Producer & Producer & Producer & Injector \\
$X$ location & 2 & 20 & 20 & 2 & 11 \\
$Y$ location & 2 & 2 & 20 & 20 & 11 \\
Constraint & $1500 \mathrm{psi}$ & $1500 \mathrm{psi}$ & $1500 \mathrm{psi}$ & $1500 \mathrm{psi}$ & $5000 \mathrm{STB} / \mathrm{D}$ \\
\hline
\end{tabular}

block. The initial reservoir pressure is 3600 psia, which is equal to the bubble point pressure of the reservoir fluid. Thus, there is no free-gas initially present inside the reservoir and initial gas saturation equals zero. Also, initial solution gas/oil ratio is $1.390 \mathrm{MSCF} / \mathrm{STB}$ and initial water saturation is 0.3 .

The synthetic reservoir is a five-spot in which there are four production wells (P1, P2, P3, and P4) at the corners of the domain and a water injection well (I1) at the center of the reservoir. Water is injected through the injection well at a constant rate of $5000 \mathrm{STB} / \mathrm{D}$ and all producers have constant bottomhole pressure of 1500 psia. Well (block) locations and specifications are listed in Table 1 . Oil, water, and gas production rates of the producers and bottom-hole pressure of the injection well are used as the production data; these provide 13 data points for each assimilation cycle. Because real historical data from the field side is not available, we have to generate synthetic historical data from the reservoir simulator using the true model parameters. Total production history is 720 days and measurements are available every 60 days; hence, we have 12 assimilation cycles. To mimic actual noisy measurements [44], a white Gaussian noise is added to the synthetic historical data. Standard deviation of this noise is 10 psi for bottom-hole pressure, $5 \mathrm{STB} / \mathrm{D}$ for oil and water production rates, and $8 \mathrm{MSCF} / \mathrm{D}$ for gas production rate.

\subsection{Reference relative permeability and capillary pressure curves}

Throughout this study, endpoint saturations are assumed to be known. We consider $S_{\text {orw }}=S_{\text {org }}=0.11$, $S_{w c}=0.152$, and $S_{g c}=0$. Conventionally, critical gas saturation $\left(S_{g c}\right)$ is assumed to be zero whenever the reservoir is initially undersaturated [25]. Similar values have been incorporated for endpoint saturations by other researchers [45].

Mean values and standard deviations of the unknown model parameters are considered as the prior knowledge (prior probability) in Table 2. Also, some typical arbitrary values are selected for the true values of these parameters. They, however, should be in the ranges covered by Gaussian distributions whose mean and standard deviations are specified in Table 2. Therefore, reference relative permeability and capillary pressure curves are generated with these true values and shown in Figures 2 and 3, respectively.
Table 2. Reference values of the relative permeability and capillary pressure parameters.

\begin{tabular}{lccc}
\hline Parameter & $\begin{array}{c}\text { Average } \\
\text { value }\end{array}$ & $\begin{array}{c}\text { Standard } \\
\text { deviation }\end{array}$ & $\begin{array}{c}\text { Reference } \\
\text { value }\end{array}$ \\
\hline$b_{0}$ & 3.00 & 0.80 & 3.70 \\
$a_{w}$ & 0.55 & 0.15 & 0.40 \\
$b_{w}$ & 5.00 & 1.00 & 4.00 \\
$P_{c o w}^{*}$ & 100.00 & 10.00 & 90.00 \\
$n_{c o w}$ & 3.50 & 0.80 & 4.30 \\
$b_{\text {og }}$ & 4.00 & 0.70 & 4.70 \\
$a_{g}$ & 0.70 & 0.20 & 0.85 \\
$b_{g}$ & 2.20 & 0.50 & 1.70 \\
$P_{c g o}^{*}$ & 50.00 & 5.00 & 55.00 \\
$n_{c g o}$ & 4.00 & 0.70 & 4.70 \\
\hline
\end{tabular}

Reference model is the simulation model in which all the unknown parameters are set to their true values, and will be used to evaluate the assimilation process. True observations are also obtained from the reservoir simulator using this reference model. Subsequently, estimated model parameters and updated observations are compared with their true values.

\section{Results and discussions}

\subsection{Scenario 1: Estimation of shape factors}

In this scenario, we assume all the endpoints to be known. Hence, there exist only 6 parameters to be estimated by assimilation of the observation data with the dynamic model:

$$
\mathbf{m}=\left[b_{o}, b_{w}, n_{c o w}, b_{o g}, b_{g}, n_{c g o}\right]^{T} .
$$

A typical number of 100 ensemble members are considered for this scenario. Each ensemble member is generated from a set of random numbers with Gaussian distribution whose mean and standard deviation are listed in Table 3. That is, a number of 100 random numbers are generated for $b_{o}$ from a normal distribution with mean and standard deviation equal to 3 and 0.8 , respectively, $\left[b_{o} \sim \mathbf{N}(3,0.8)\right]$. The same procedure is repeated for $b_{w}, n_{\text {cow }}, b_{o g}, b_{g}$, and $n_{c g o}$ using their own mean and standard deviation values. This provides a number of 100 different realizations for the model parameters, which are then used in the initial ensemble. 
Table 3. Initial, estimated, and reference values of the relative permeability and capillary pressure parameters in Scenario 1.

\begin{tabular}{cccccccc}
\hline Parameter & $\begin{array}{c}\text { Average } \\
\text { value }\end{array}$ & STD & $\begin{array}{c}\text { Reference } \\
\text { value }\end{array}$ & $\begin{array}{c}\text { Average } \\
\text { initial }\end{array}$ & $\begin{array}{c}\text { Estimated } \\
\text { value }\end{array}$ & $\begin{array}{c}\text { Final } \\
\text { STD }\end{array}$ & $\begin{array}{c}\text { Relative } \\
\text { error }(\%)\end{array}$ \\
\hline$b_{o}$ & 3.00 & $8.00 \times 10^{-1}$ & 3.70 & 3.06 & 3.70 & $1.36 \times 10^{-2}$ & 0.00 \\
$b_{w}$ & 5.00 & $1.00 \times 10^{0}$ & 4.00 & 5.16 & 4.00 & $1.42 \times 10^{-2}$ & 0.00 \\
$n_{\text {cow }}$ & 3.50 & $8.00 \times 10^{-1}$ & 4.30 & 3.67 & 4.31 & $5.00 \times 10^{-2}$ & 0.23 \\
$b_{\text {og }}$ & 4.00 & $7.00 \times 10^{-1}$ & 4.70 & 3.97 & 4.67 & $2.57 \times 10^{-1}$ & 0.64 \\
$b_{g}$ & 2.20 & $5.00 \times 10^{-1}$ & 1.70 & 2.19 & 1.70 & $1.57 \times 10^{-2}$ & 0.00 \\
$n_{\text {cgo }}$ & 4.00 & $7.00 \times 10^{-1}$ & 4.70 & 3.94 & 4.64 & $4.32 \times 10^{-1}$ & 1.28 \\
\hline
\end{tabular}

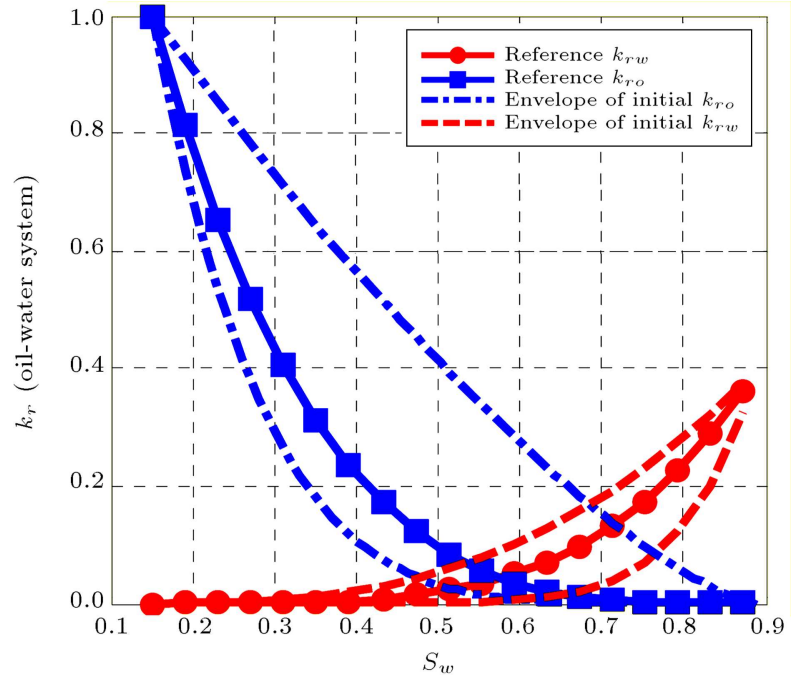

(a)

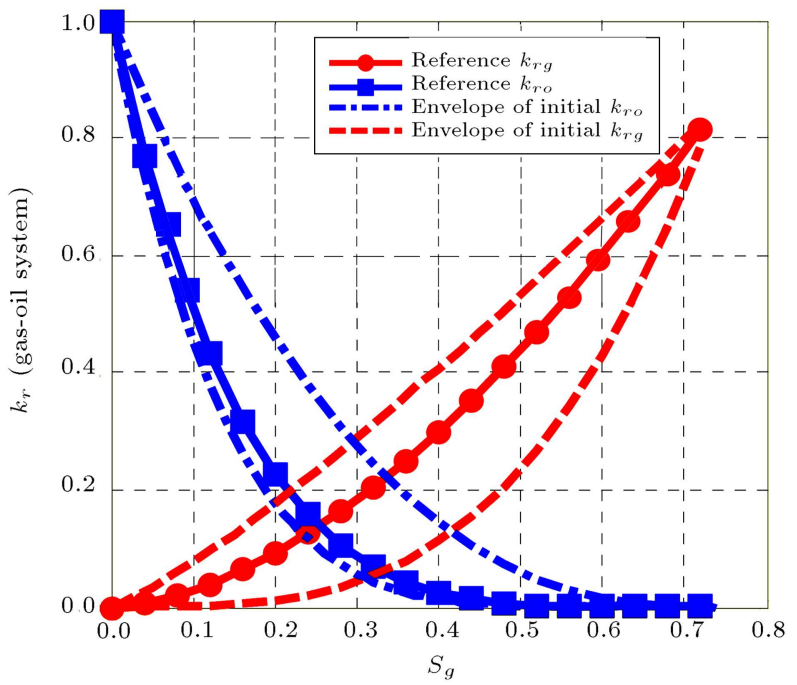

(b)

Figure 2. Reference relative permeabilities and envelopes of initial ensembles for (a) the oil-water system and (b) the gas-oil system.

The boundary envelopes of the initial ensemble members are depicted in Figures 2 and 3 . These envelopes are generated with the maximum and minimum values of the ensemble members and all the other initial curves will be within the range covered by these

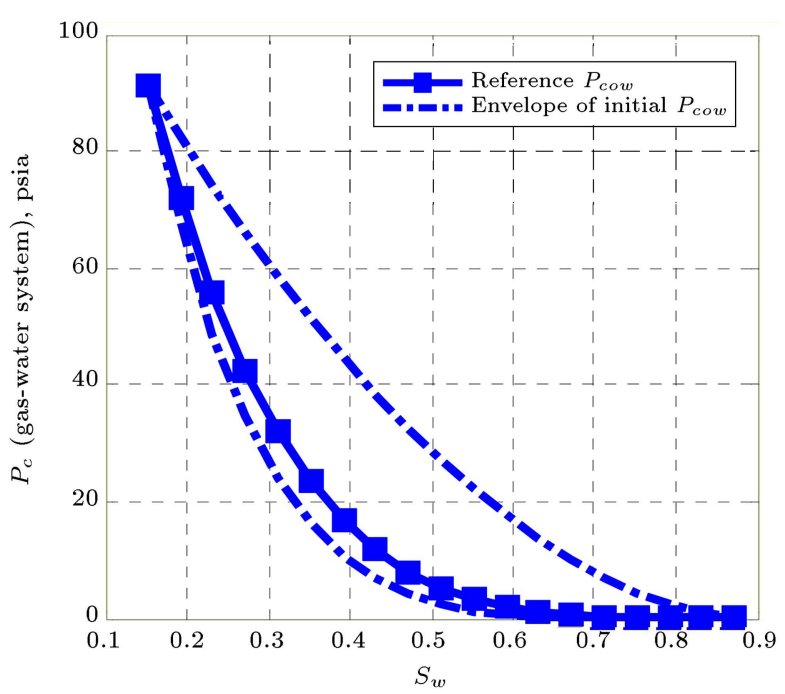

(a)

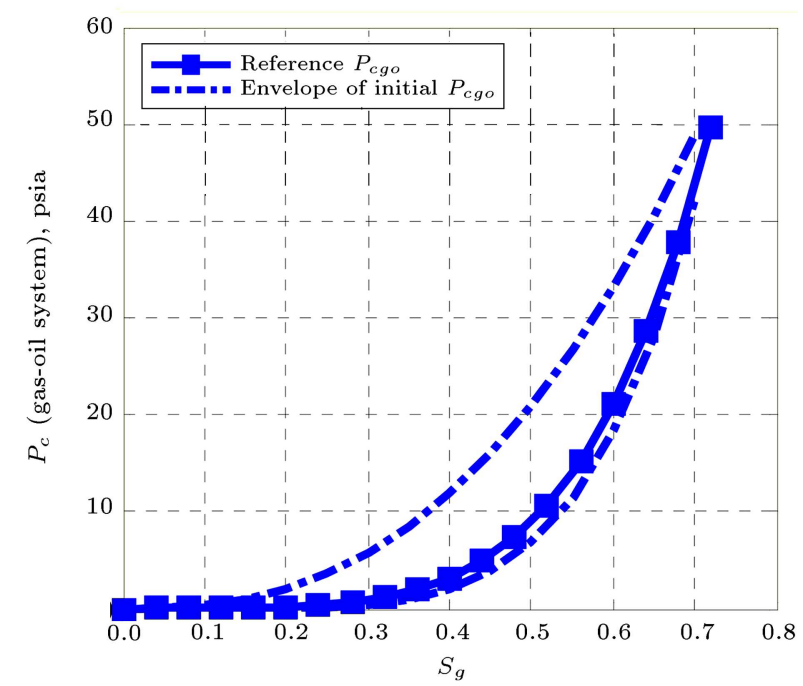

(b)

Figure 3. Reference capillary pressure and envelopes of initial ensembles for (a) the oil-water system and (b) the gas-oil system.

envelopes. As all of the endpoints (i.e., $a_{w}, P_{\text {cow }}^{*}, a_{g}$, $\left.P_{c g o}^{*}\right)$ are assumed to be known and constant in this scenario, there is no spread at the endpoints of the curves.

The averages of the estimated relative permeabil- 


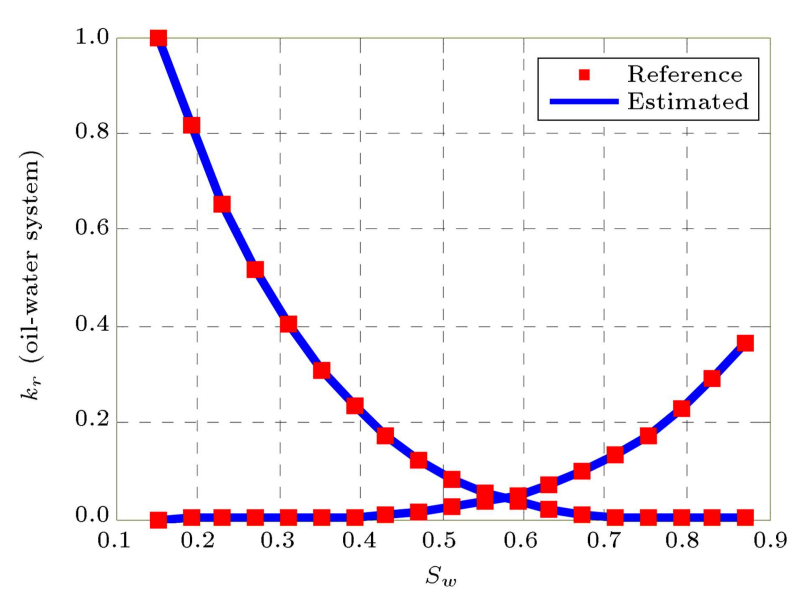

(a)

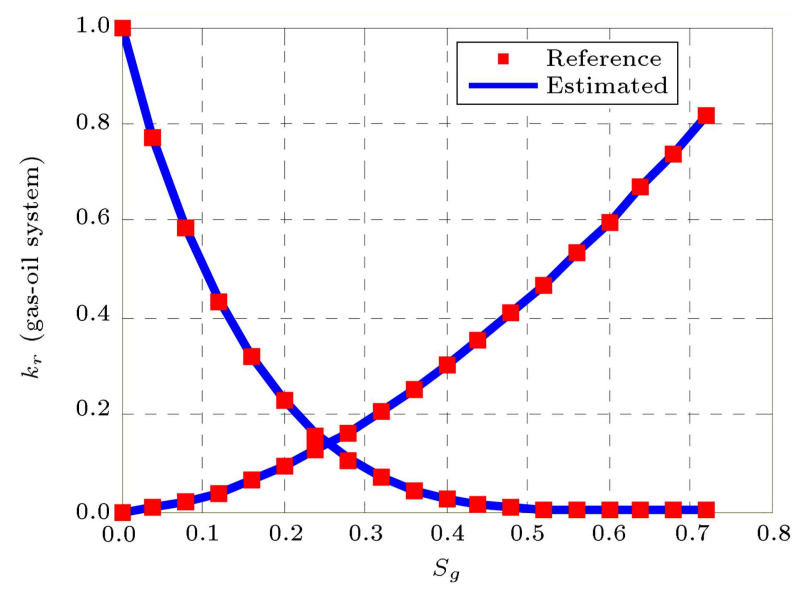

(b)

Figure 4. Estimated and reference relative permeability curves for (a) the oil-water system and (b) the gas-oil system in Scenario 1.

ity and capillary pressure curves are compared with their corresponding references in Figures 4 and 5 . Clearly, an accurate evaluation of the relative permeabilities and capillary pressures is performed with the maximum relative error of $1.28 \%$. The initial, estimated, and reference parameters are listed in Table 3.

Figure 6 displays evolution of the relative permeability and capillary pressure parameters versus assimilation time. The dots represent mean values calculated by averaging ensemble members and error bars show standard deviations. Before the assimilation, there is a large error and uncertainty for all the parameters. As assimilation proceeds, parameters get closer to their reference values while standard deviations (uncertainties) of the updated parameters are usually reduced. This is in agreement with other investigations in relation to the estimation of the relative permeabilities and capillary pressures $[11,21,24]$.

Although the uncertainties in the parameters $b_{o}$, $b_{w}, n_{\text {cow }}$, and $b_{g}$ have been reduced significantly in Figure 6 , the shape factors of the capillary pressure curve and oil relative permeability in the gas-oil system

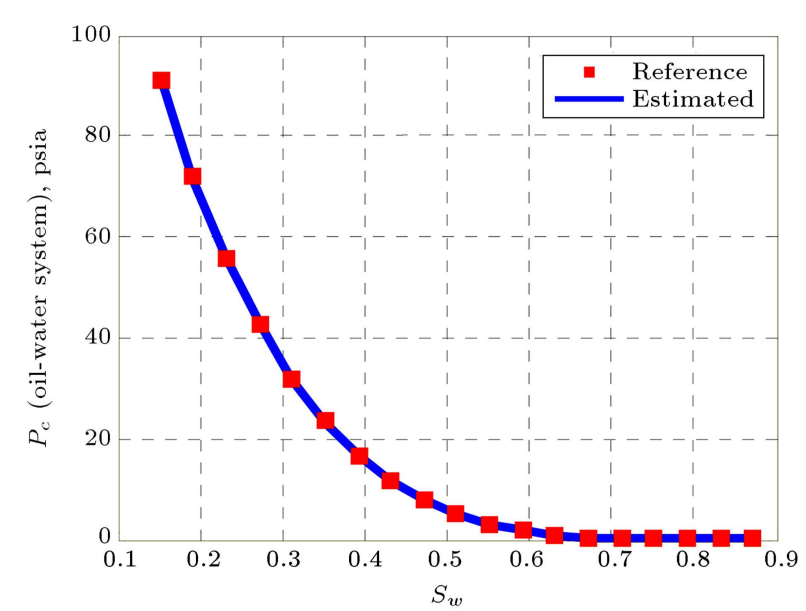

(a)

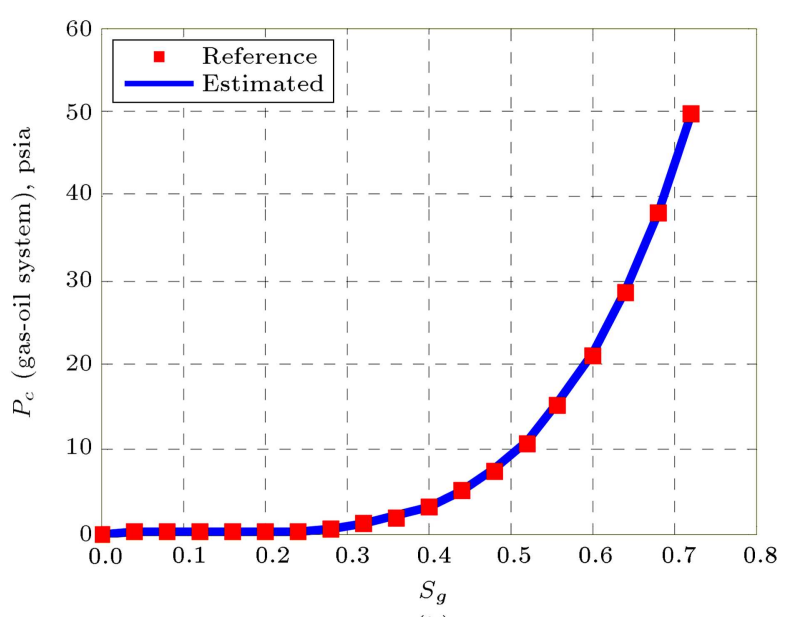

(b)

Figure 5. Estimated and reference capillary pressure curves for (a) the oil-water system and (b) the gas-oil system in Scenario 1.

$\left(n_{c g o}\right.$ and $\left.b_{o g}\right)$ still have large uncertainty. Actually, for the most sensitive parameters, better estimation and lower uncertainty are obtained during the assimilation process. Thus, it can be inferred that parameters $b_{o}$, $b_{w}, n_{\text {cow }}$ and $b_{g}$ have more profound effects on the fluid flow through porous media and the production data. Furthermore, larger uncertainty and larger relative error for the estimation of $n_{c g o}$ and $b_{o g}$ may have root in the fact that production data contain information reflecting low free gas saturation inside the reservoir. Figure 7 accentuates that maximum free-gas saturation in the reservoir for all ensemble members does not exceed 0.18 .

Moreover, in Section 4.3, the sensitivity of the observation data to the unknown model parameters will be discussed thoroughly and it will be illustrated that estimation of the most sensitive parameters is closer to their reference values and, also, uncertainty of these parameters is decreased significantly at the end of assimilation.

Average initial values of the relative permeability 


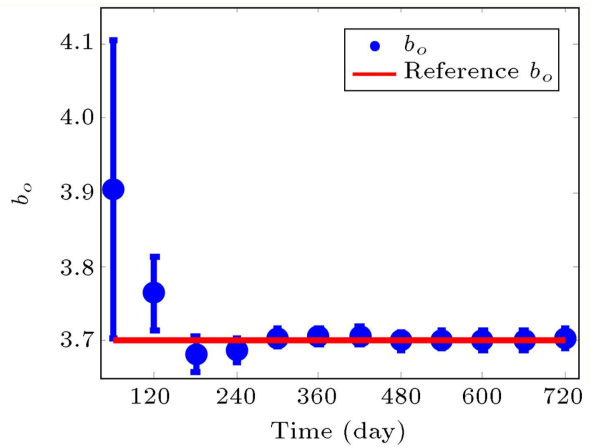

(a)

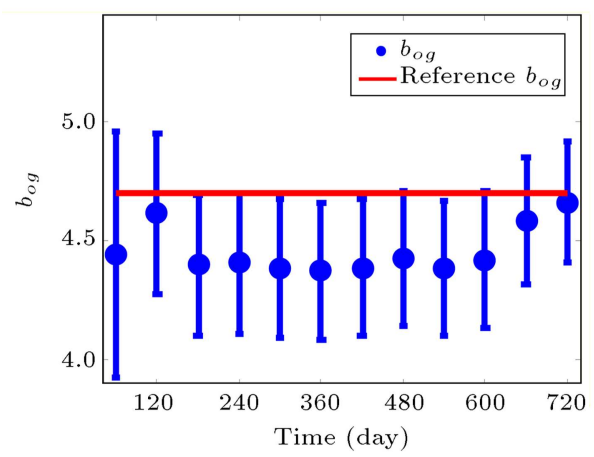

(c)

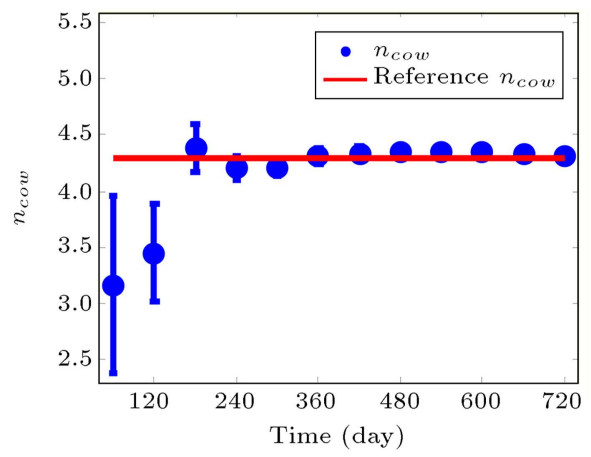

(e)

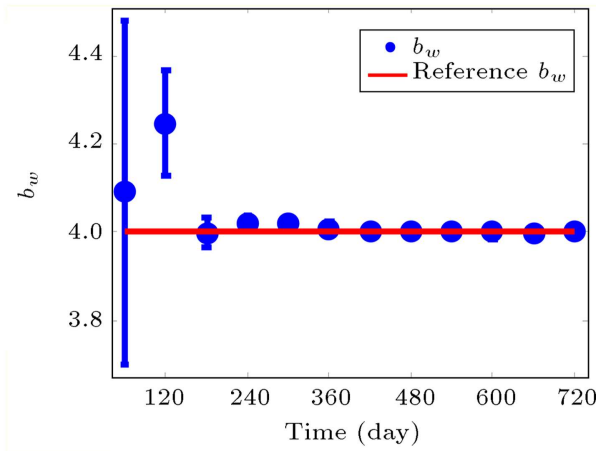

(b)

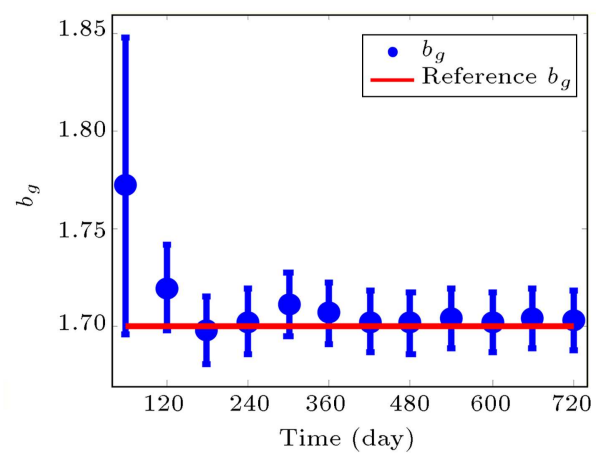

(d)

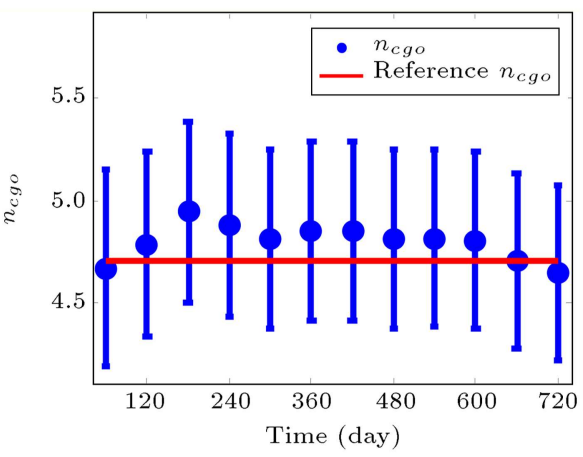

(f)

Figure 6. Estimation of the relative permeability and capillary pressure parameters versus assimilation time: (a) $b_{o}$, (b) $b_{w}$, (c) $b_{o g}$, (d) $b_{g}$, (e) $n_{\text {cow }}$, and (f) $n_{c g o}$, in Scenario 1.

and capillary pressure parameters are listed in Table 3 , which are relatively close to the mean values of their distributions. For the first assimilation cycle in Figure 6 , parameters $b_{o}, b_{w}$, and $b_{g}$ are overestimated while the other parameters are underestimated relative to their corresponding reference values. This observation can be better justified using the Kalman filter equation (Eq. (10)), in which the assimilated ensemble mean is equal to the forecasted ensemble mean plus the update term, $\mathbf{K}\left(\mathbf{d}-\mathbf{H} \mathbf{x}^{f}\right)$. This term makes the model parameters adjust in some way that closer agreement between the predicted observations and real measurements is obtained when assimilated parameters are utilized in the reservoir simulator. Thus, any increase or decrease in the assimilated model parameters is interpreted through the update term in the Kalman filter equation.

Figure 8 shows the trend of RRMSE variation, as calculated by Eq. (19), versus assimilation time. It is a decreasing trend, which means that the overall estimation error is reduced as the assimilation proceeds. Results of the history matching process are presented in Figure 9. At each assimilation cycle, updated ensemble of model parameters is used to generate its corresponding relative permeability and capillary pressure curves. Then, reservoir simulator is re-run with the updated curves to calculate oil, gas, and water production rates for all the producers and the bottom-hole pressure of the injector. Also, before the assimilation, initial ensemble members were used in the simulation model to forecast reservoir performance and assess the uncertainty. The gray and the black curves denote the results from the initial and updated ensembles, respectively, and the red line represents the true observations from the reference model. The 


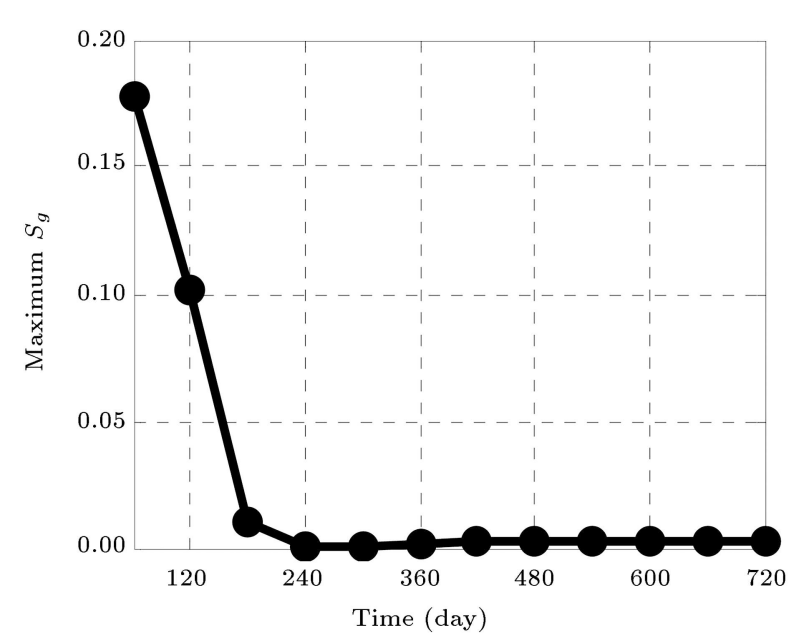

Figure 7. Maximum free gas saturation in the reservoir versus assimilation time.

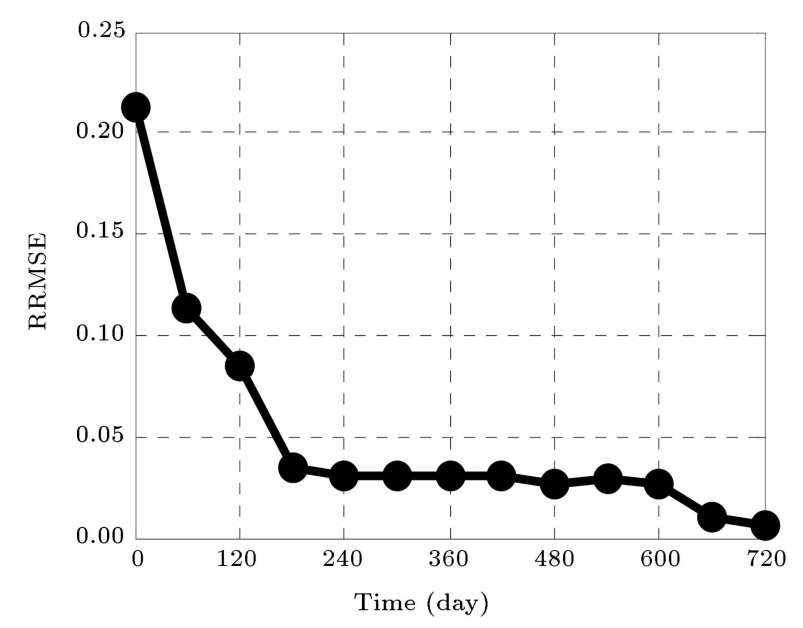

Figure 8. Relative root-mean-square error versus assimilation time for Scenario 1.

updated ensemble members perform fairly well and closely match the true observations for different types of production data.

Before the breakthrough of water, no water is produced from the production wells and, thus, there is no data of water production. However, water is injected via the injection well throughout the production history. Bottom-hole pressure of the injection well is internally related to the relative permeability and capillary pressure curves through the simulation equations. As the bottom-hole pressure of the injection well is also included in the historical data used for the assimilation process, it will provide enough information for estimating parameters of water flow functions during the pre-breakthrough period.

\subsection{Scenario 2: Estimation of shape factors and endpoints}

When the endpoints are also unknown, there exist 10 parameters to be estimated by assimilation of the observation data with the dynamic model, which are shown as vector $\mathbf{m}$ in Eq. (17). This makes the history matching problem more complicated and challenging due to its higher degree of freedom. Apparently, this requires larger number of ensemble members to make the filter converge to appropriate estimates. If the ensemble size is not large enough to approximate the prior covariance properly, the ensemble members may collapse to a single one or the updated ensemble members may get improperly tuned with the observation data; either case results in a poor data match and, thus, filter divergence occurs. As Figure 10 depicts, using a number of 100 ensemble members $(N=100)$, similar to what used previously, led to the divergence of the filter. This is despite the fact that RRSME was decreasing at the early assimilation cycles; however, after a while, RRMSE started to increase, which ultimately resulted in filter divergence. Subsequently, a twice larger ensemble size was examined $(N=200)$, which made the filter converge and RRMSE decrease along the assimilation time. The same approach is implemented by some other researchers, such as $\mathrm{Li}$ et al. [26], in order to determine the appropriate large-enough ensemble size. Additionally, to our knowledge, no researchers have provided an analytical error analysis to deterministically indicate bounds on the number of realizations required for convergence, and this is certainly application-dependent.

Each ensemble is generated from a Gaussian distribution with the mean and standard deviation values listed in Table 4. As with the first scenario, the averages of the estimated relative permeability and capillary pressure curves have been compared with their corresponding reference values in Figures 11 and 12, respectively. Obviously, more accurate estimation of the relative permeability and capillary pressure curves is obtained for the oil-water system. This will be justified by calculation of dimensionless sensitivities in Section 4.3. For the endpoint of water and gas relative permeabilities, cut-off values of $a_{w}=1.0$, and $a_{g}=1.0$ have been used and all the greater values are set to 1.0 during simulation.

Except for $P_{c g o}^{*}$, the estimations of the other unknown parameters at the end of assimilation are much closer to their corresponding reference values than to the initial ones. The initial, estimated, and reference parameters are also listed in Table 4 . The estimation error after the assimilation is small enough and its maximum relative error is about $8.89 \%$.

Figures 13 and 14 represent evolution of the relative permeability and capillary pressure parameters versus assimilation time for the oil-water and gas-oil systems, respectively. Before the assimilation, there is a large error and large uncertainty for all the parameters. During the assimilation, model parameters get closer to their reference values except for $P_{c g o}^{*}$ in which the initial and estimated values are almost the same 

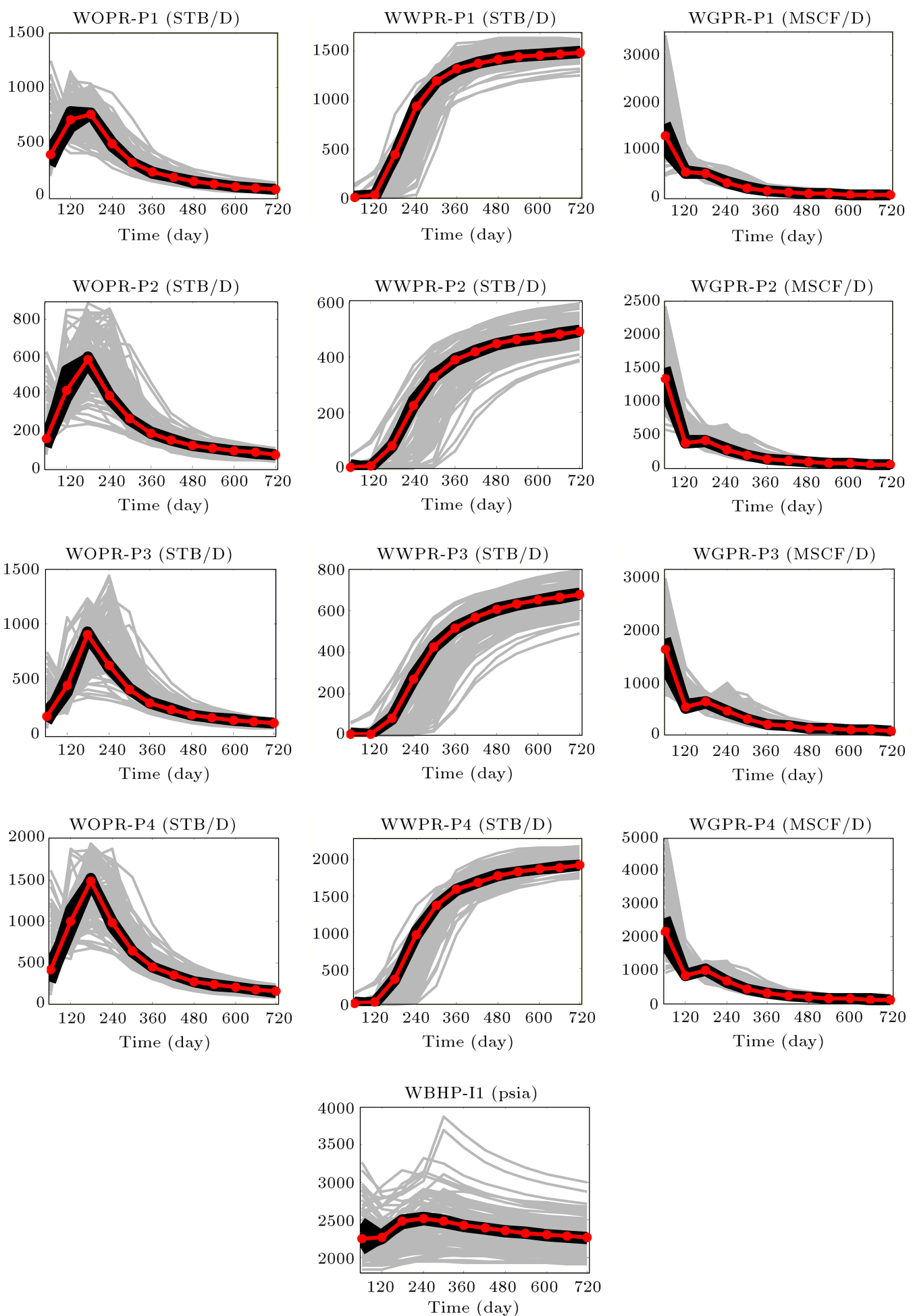

Figure 9. History matching results for oil production rate, water production rate, gas production rate, and bottom-hole pressure in Scenario 1.

(see Table 4). Furthermore, estimation of $n_{c g o}$ has been only improved at the first assimilation cycle, while no improvement is achieved during the next cycles. In general, estimation of the most sensitive parameters is closer to their reference values and uncertainty of these parameters is decreased significantly at the end of assimilation. Therefore, it can be inferred that parameters $b_{o}, a_{w}, b_{w}, n_{\text {cow }}$, and $b_{g}$ have more profound effects on the production data. Later, the sensitivity of the observation data to the unknown parameters will be discussed. Also, the larger relative error for the estimation of the relative permeabilities and capillary 
Table 4. Initial, estimated, and reference values of the relative permeability and capillary pressure parameters in Scenario 2.

\begin{tabular}{ccccccccc}
\hline Parameter & $\begin{array}{c}\text { Average } \\
\text { value }\end{array}$ & STD & $\begin{array}{c}\text { Reference } \\
\text { value }\end{array}$ & $\begin{array}{c}\text { Average } \\
\text { initial }\end{array}$ & $\begin{array}{c}\text { Estimated } \\
\text { value }\end{array}$ & $\begin{array}{c}\text { Final } \\
\text { STD }\end{array}$ & $\begin{array}{c}\text { STD } \\
\text { ratio* }\end{array}$ & $\begin{array}{c}\text { Relative } \\
\text { error (\%) }\end{array}$ \\
\hline$b_{o}$ & 3.00 & $8.00 \times 10^{-1}$ & 3.70 & 3.01 & 3.71 & $9.10 \times 10^{-3}$ & $1.14 \times 10^{-2}$ & 0.27 \\
$a_{w}$ & 0.55 & $1.50 \times 10^{-1}$ & 0.40 & 0.55 & 0.40 & $4.40 \times 10^{-3}$ & $2.93 \times 10^{-2}$ & 0.00 \\
$b_{w}$ & 5.00 & $1.00 \times 10^{0}$ & 4.00 & 5.07 & 3.99 & $3.73 \times 10^{-2}$ & $3.73 \times 10^{-2}$ & 0.25 \\
$P_{c o w}^{*}$ & 100.00 & $1.00 \times 10^{1}$ & 90.00 & 98.79 & 89.56 & $3.97 \times 10^{0}$ & $3.97 \times 10^{-1}$ & 0.49 \\
$n_{\text {cow }}$ & 3.50 & $8.00 \times 10^{-1}$ & 4.30 & 3.50 & 4.29 & $6.00 \times 10^{-2}$ & $7.50 \times 10^{-2}$ & 0.23 \\
$b_{\text {og }}$ & 4.00 & $7.00 \times 10^{-1}$ & 4.70 & 3.96 & 4.95 & $3.18 \times 10^{-1}$ & $4.54 \times 10^{-1}$ & 5.31 \\
$a_{g}$ & 0.70 & $2.00 \times 10^{-1}$ & 0.85 & 0.71 & 0.88 & $5.74 \times 10^{-2}$ & $2.87 \times 10^{-1}$ & 3.53 \\
$b_{g}$ & 2.20 & $5.00 \times 10^{-1}$ & 1.70 & 2.22 & 1.73 & $3.72 \times 10^{-2}$ & $7.44 \times 10^{-2}$ & 1.76 \\
$P_{c g o}^{*}$ & 50.00 & $5.00 \times 10^{0}$ & 55.00 & 50.02 & 50.11 & $4.83 \times 10^{0}$ & $9.66 \times 10^{-1}$ & 8.89 \\
$n_{\text {cgo }}$ & 4.00 & $7.00 \times 10^{-1}$ & 4.70 & 4.03 & 4.91 & $4.98 \times 10^{-1}$ & $7.11 \times 10^{-1}$ & 4.47 \\
\hline
\end{tabular}

*: Ratio of the final STD to the initial std.

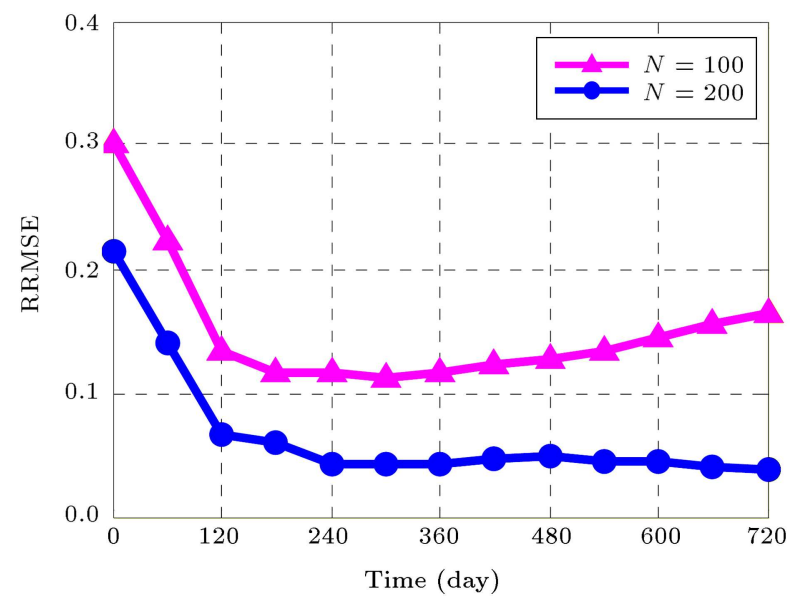

Figure 10. Relative root-mean-square error versus assimilation time for Scenario 2.

pressure of the gas-oil system may be due to the fact that production data contains information reflecting low free-gas saturation (see Figure 7).

Similar to Figure 9, at each assimilation cycle, reservoir simulator is re-run with the updated relative permeability and capillary pressure curves to calculate oil, gas, and water production rates for all the producers and the bottom-hole pressure of the injector. In Figure 15, the gray and the black curves denote the results from the initial and updated ensembles, respectively; and the red line represents the true observations from the reference model. It is clear that updated ensemble members closely match the true observations for different types of production data and this further verifies that relative permeabilities and capillary pressures are well adjusted during assimilation using the EnKF.

\subsection{Sensitivity analysis}

In this section, the sensitivity of the observation data to the relative permeability and capillary pressure

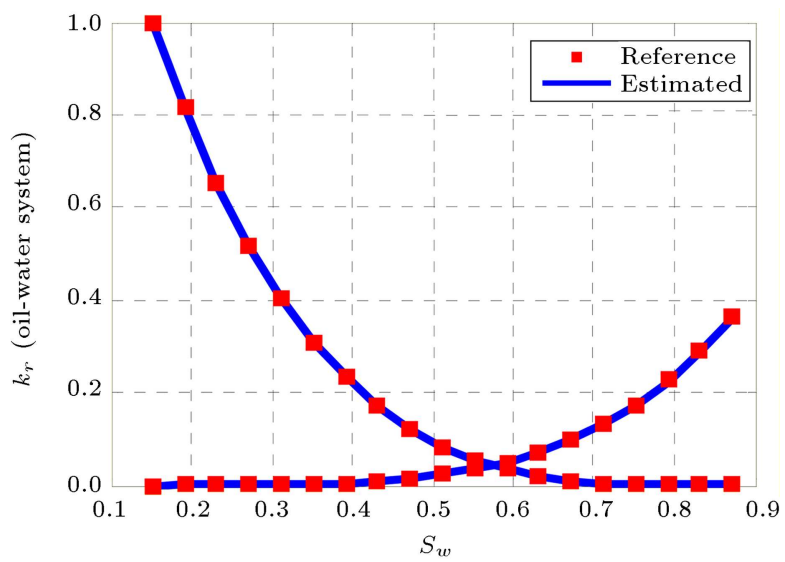

(a)

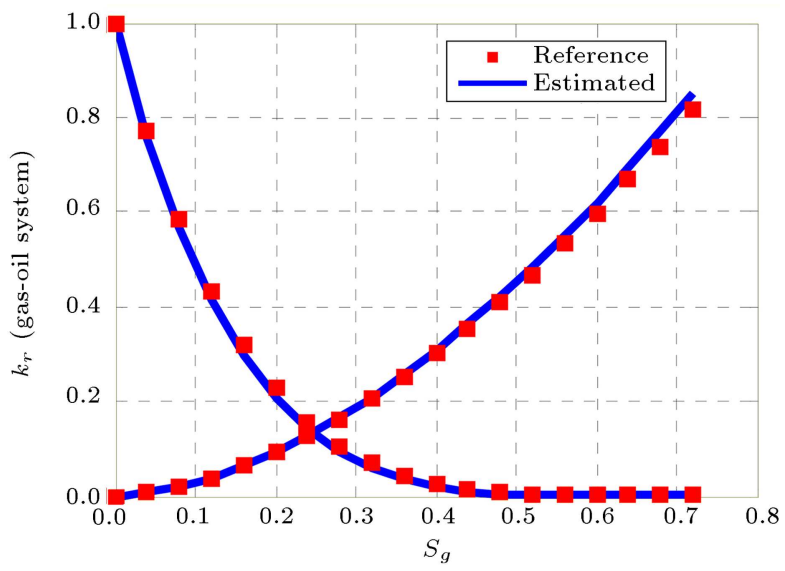

(b)

Figure 11. Estimated and reference relative permeability curves for (a) the oil-water system and (b) the gas-oil system in Scenario 2.

parameters obtained in the second scenario is analyzed. Dimensionless sensitivity coefficients provide a relative measure of how different data affect estimates of the model parameters. Dimensionless sensitivity of observation $d_{i}$ to model parameter $m_{j}$ is given by $[45,46]$ : 


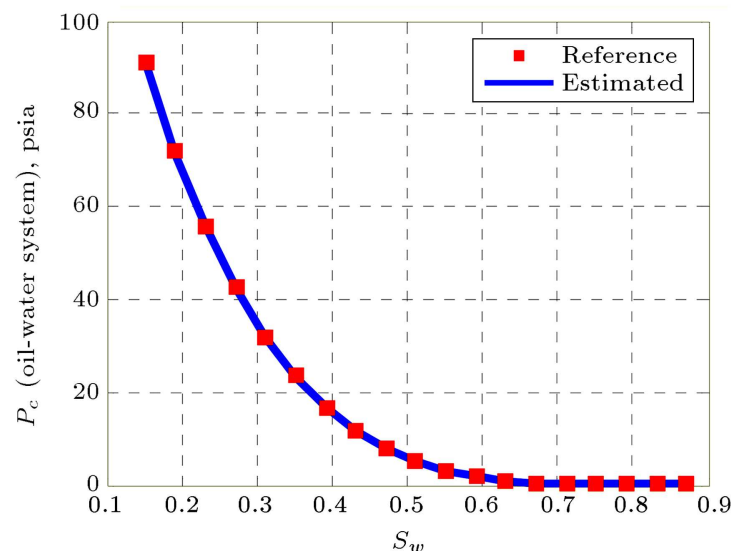

(a)

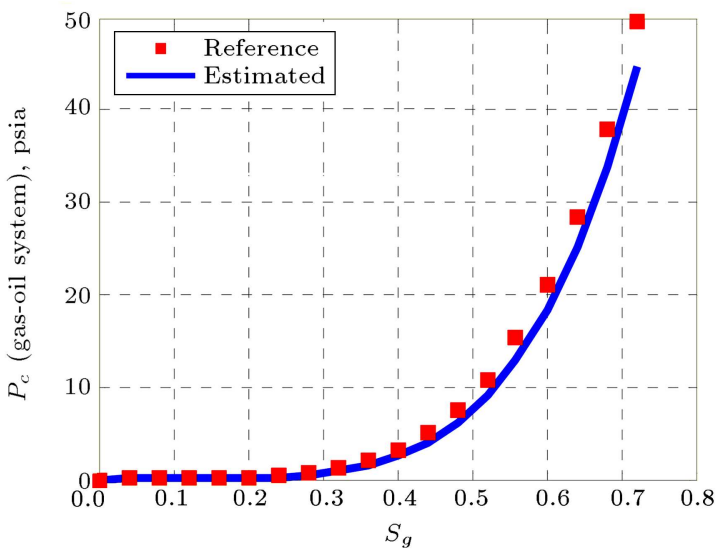

(b)

Figure 12. Estimated and reference capillary pressure curves for (a) the oil-water system and (b) the gas-oil system in Scenario 2.

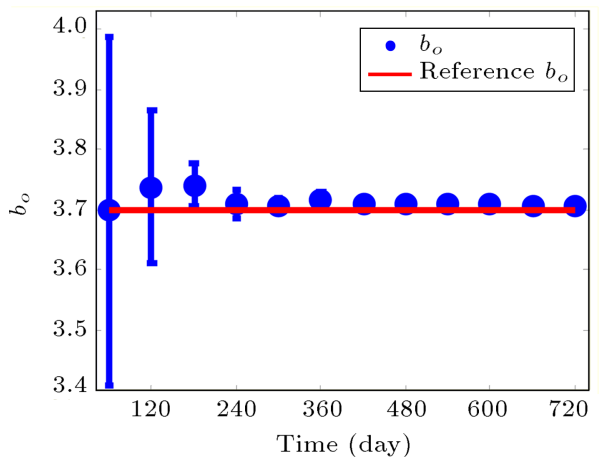

(a)

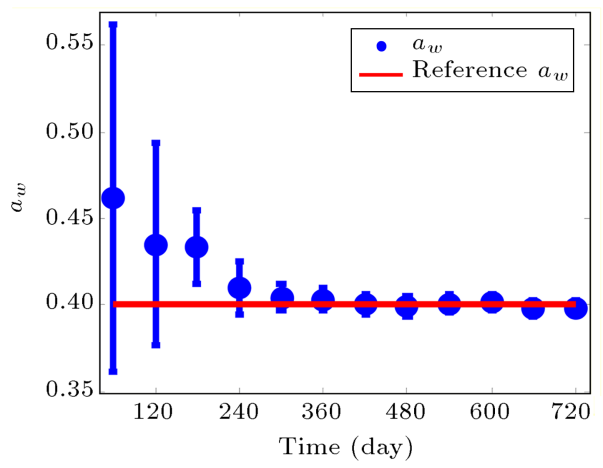

(c)

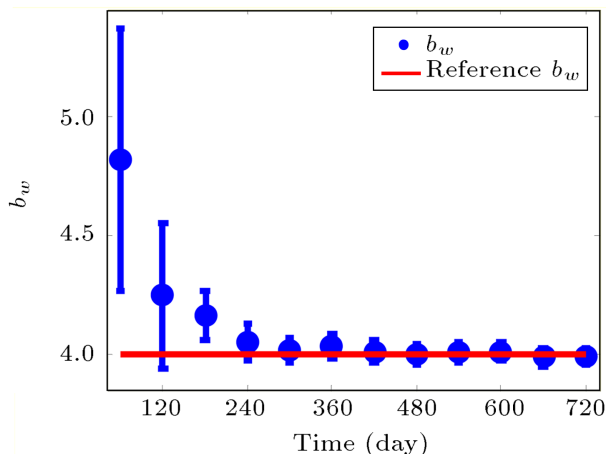

(b)

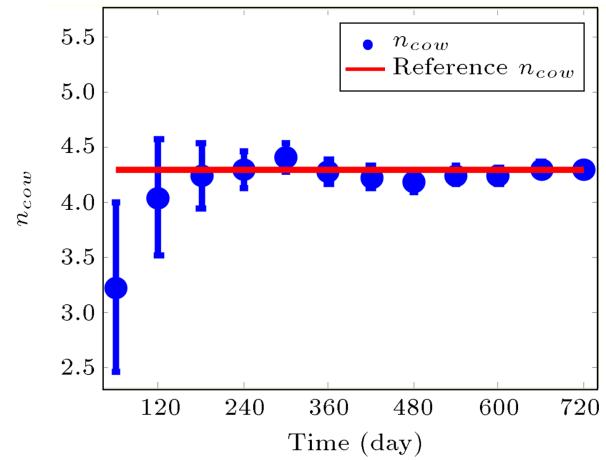

(d)

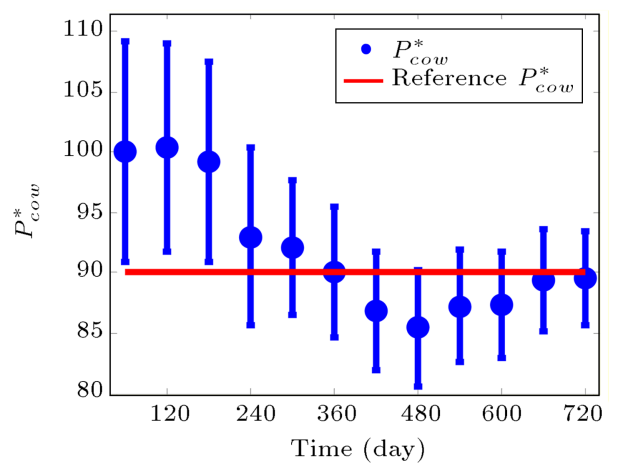

(e)

Figure 13. Estimation of the relative permeability and capillary pressure parameters versus assimilation time for the oil-water system: (a) $b_{o}$, (b) $b_{w}$, (c) $a_{w}$, (d) $n_{\text {cow }}$, and (e) $P_{c o w}^{*}$, in Scenario 2. 


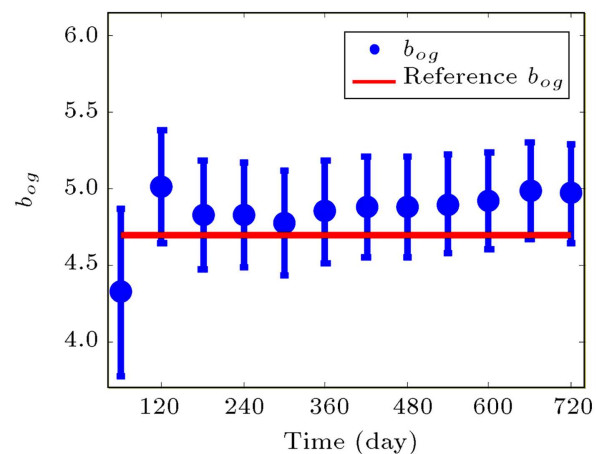

(a)

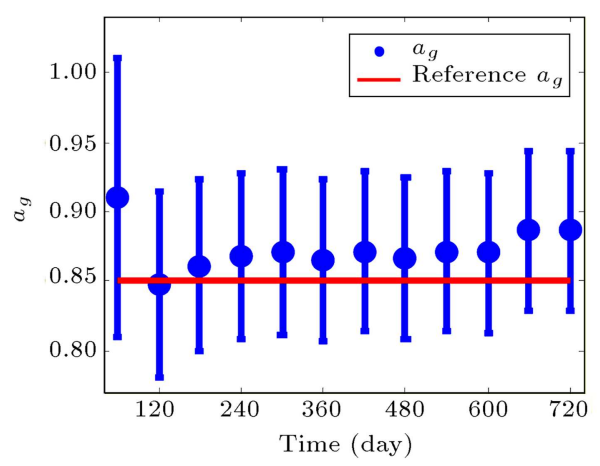

(c)

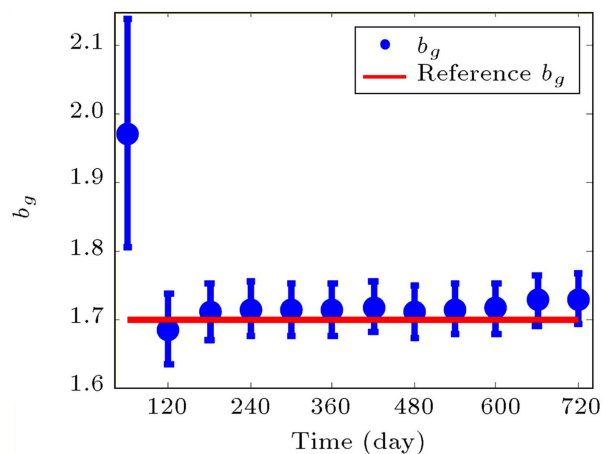

(b)

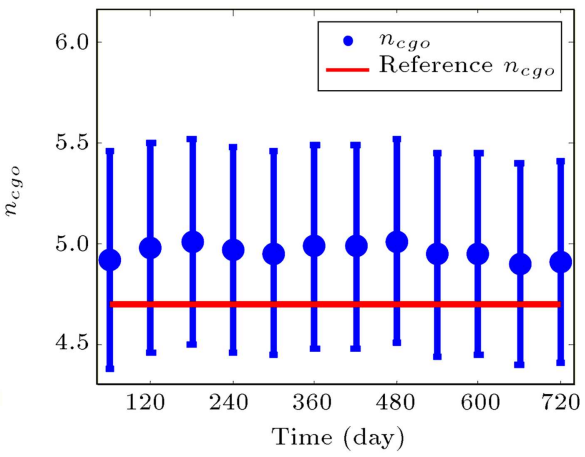

(d)

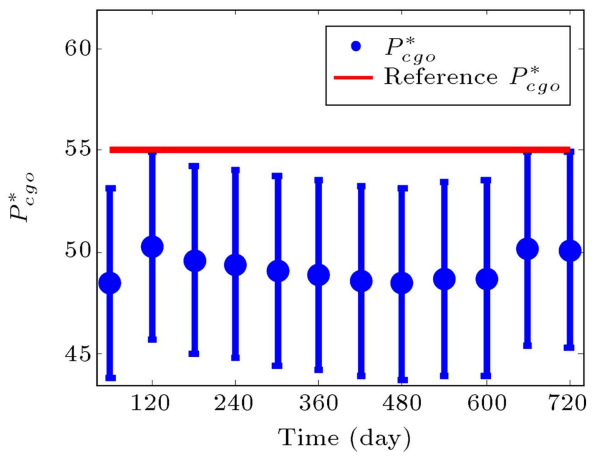

(e)

Figure 14. Estimation of the relative permeability and capillary pressure parameters versus assimilation time for the gas-oil system: (a) $b_{o g}$, (b) $b_{g}$, (c) $a_{g}$, (d) $n_{c g o}$, and (e) $P_{c g o}^{*}$, in Scenario 2.

$$
S_{i, j}=\frac{\partial d_{i}}{\partial m_{j}} \frac{\sigma_{m_{j}}}{\sigma_{d_{i}}}
$$

wherein $\sigma_{m_{j}}^{2}$ is the prior variance of model parameter $m_{j}$ and $\sigma_{d_{i}}^{2}$ is the variance of measurement error for the $i$ th observation. The derivatives involved in Eq. (21) are calculated using a five-point stencil as [47]:

$$
\frac{\partial d_{i}}{\partial m_{j}}=\frac{-f_{i, 1}+8 f_{i, 2}-8 f_{i, 3}+f_{i, 4}}{12 \Delta m_{j}},
$$

in which:

$$
\begin{aligned}
& f_{i, 1}=d_{i} \\
& \mathbf{m}=\left[m_{1}, \cdots, m_{j-1}, m_{j}+2 \Delta m_{j}, m_{j+1}, \cdots, m_{N_{p}}\right]^{T}, \\
& f_{i, 2}=d_{i} \quad \text { at }
\end{aligned}
$$

$$
\begin{aligned}
& \mathbf{m}=\left[m_{1}, \cdots, m_{j-1}, m_{j}+\Delta m_{j}, m_{j+1}, \cdots, m_{N_{p}}\right]^{T}, \\
& f_{i, 3}=d_{i} \quad \text { at } \\
& \mathbf{m}=\left[m_{1}, \cdots, m_{j-1}, m_{j}-\Delta m_{j}, m_{j+1}, \cdots, m_{N_{p}}\right]^{T}, \\
& f_{i, 4}=d_{i} \quad \text { at } \\
& \mathbf{m}=\left[m_{1}, \cdots, m_{j-1}, m_{j}-2 \Delta m_{j}, m_{j+1}, \cdots, m_{N_{p}}\right]^{T},
\end{aligned}
$$

and $\Delta m_{j}=0.01 m_{j} \cdot f_{i, 1}$ through $f_{i, 4}$ are evaluated using the reservoir simulator.

Actually, higher values of dimensionless sensitivity result in better estimation of the model parameters and lower uncertainty (lower variance) at the end of 

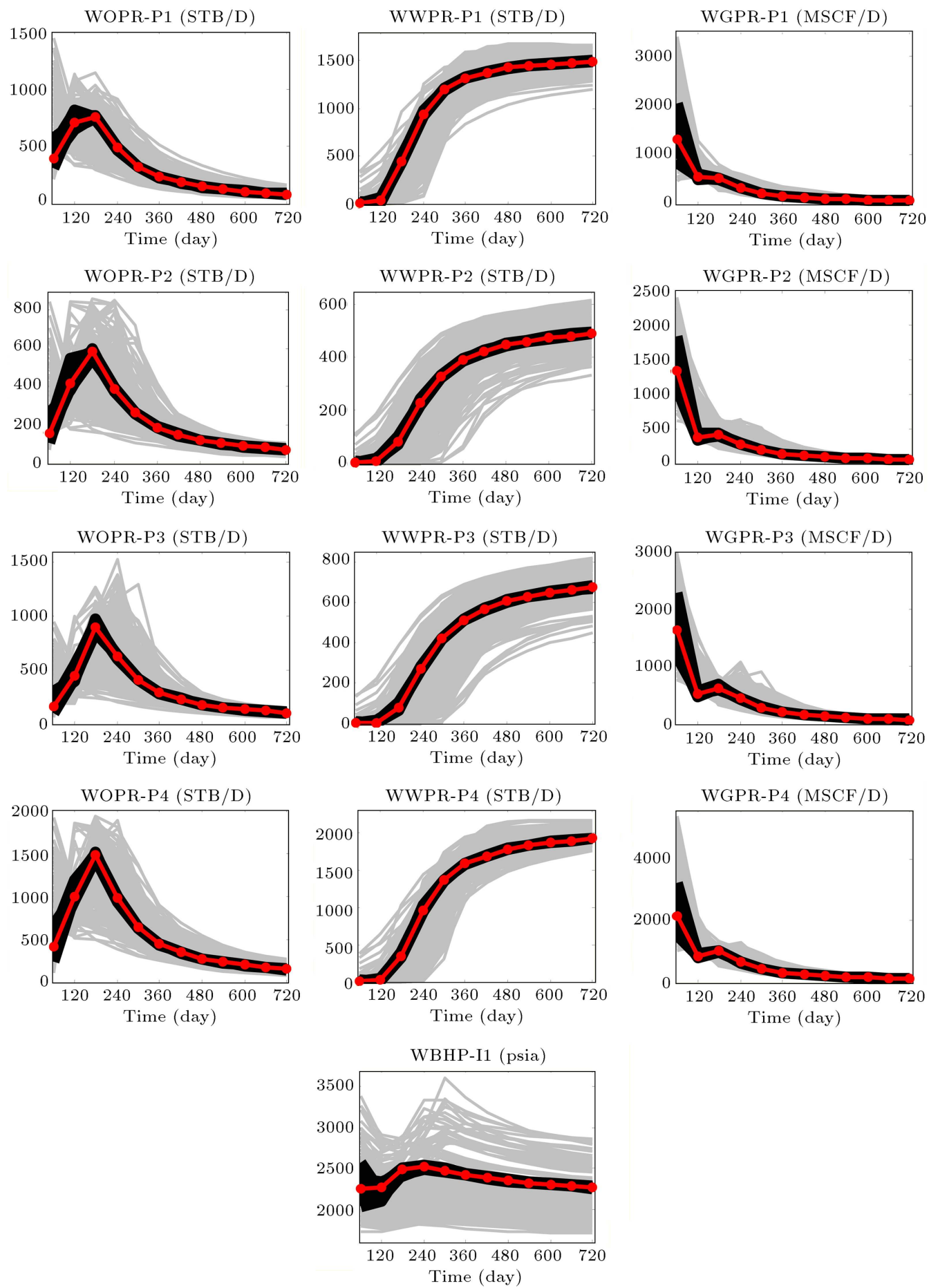

Figure 15. History matching results for oil production rate, water production rate, gas production rate, and bottom-hole pressure in Scenario 2.

assimilation. For example, if $S_{i, j}>S_{k, j}$, then use of $d_{i}$ for history matching gives a better estimate of $m_{j}$ than the one obtained by history matching based on $d_{k}$ [45].

In this section, we choose production rates of well P1 and bottom-hole pressure of well I1 so as to perform the sensitivity analysis. Figure 16 shows the dimensionless sensitivity of the oil production rate at well $\mathrm{P} 1$ to the model parameters as a function of time. It shows that oil production rate at well $\mathrm{P} 1$ is very sensitive to the parameters defining the oil-water relative permeabilities $\left(b_{o}, b_{w}\right.$, and $\left.a_{w}\right)$. Furthermore, water and gas production rates at well P1 (Figures 17 and 18) and bottom-hole pressure at well I1 (Figure 19) are quite sensitive to the oil-water relative permeability parameters. Therefore, one expects a close agreement between the estimation of the oil-water relative perme- 


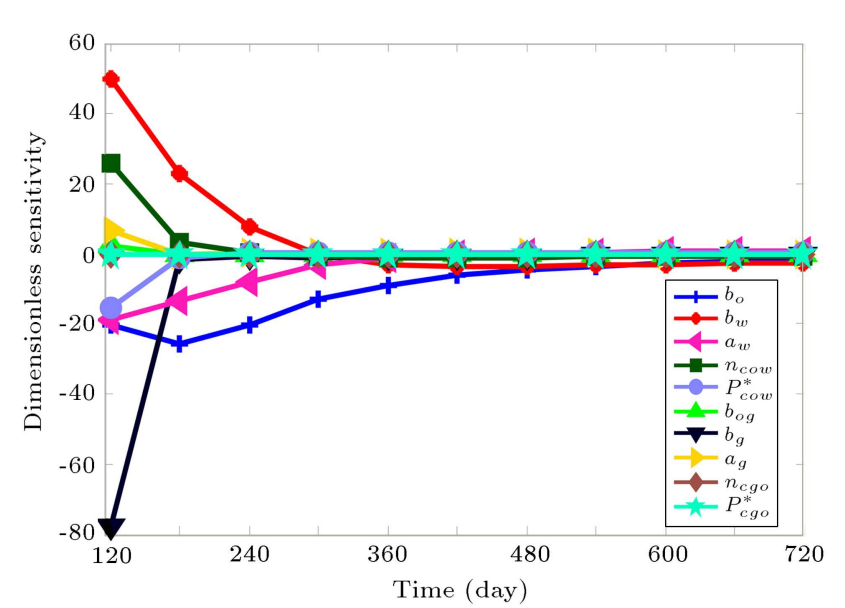

Figure 16. Dimensionless sensitivity of well P1 oil production rate to relative permeability and capillary pressure parameters.

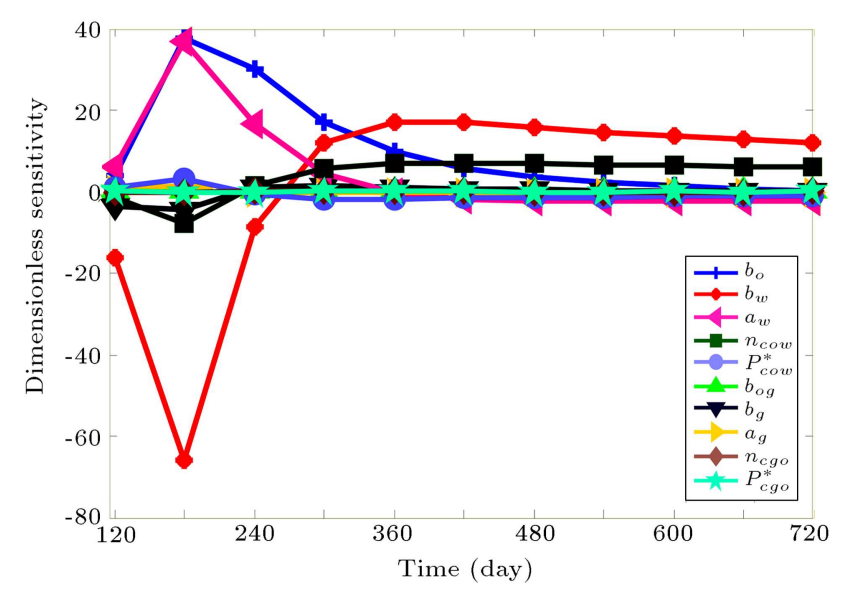

Figure 17. Dimensionless sensitivity of well P1 water production rate to relative permeability and capillary pressure parameters.

ability parameters and the reference values with low uncertainty at the end of assimilation. This is verified by the results presented in Figure 13 and Table 4. Oil production rate at early times is also sensitive to the shape factor of the gas relative permeability, $b_{g}$.

Figure 17 represents the dimensionless sensitivity coefficients of water production rate at well $\mathrm{P} 1$. As it is shown, water production is sensitive to the parameters $b_{o}, b_{w}, a_{w}$, and $n_{\text {cow }}$. Figure 18 shows that at the early times of assimilation, gas production rate of well $\mathrm{P} 1$ is quite sensitive to $b_{g}$, as expected. However, as assimilation cycle proceeds, when the free-gas saturation decreases and the gas production rate is reduced significantly, only $b_{o}, b_{w}$, and $a_{w}$ dominate the gas flow rate.

Figure 19 represents the dimensionless sensitivity coefficients of flowing bottom-hole pressure at well I1. Bottom-hole flowing pressure is strongly influenced by total mobility [45], which is the sum of the mobilities of each phase and is defined as:

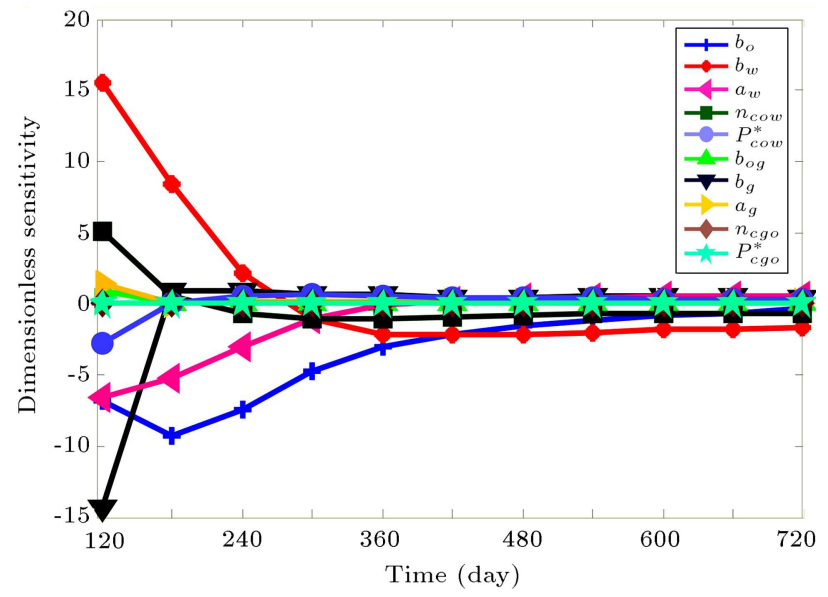

Figure 18. Dimensionless sensitivity of well P1 gas production rate to relative permeability and capillary pressure parameters.

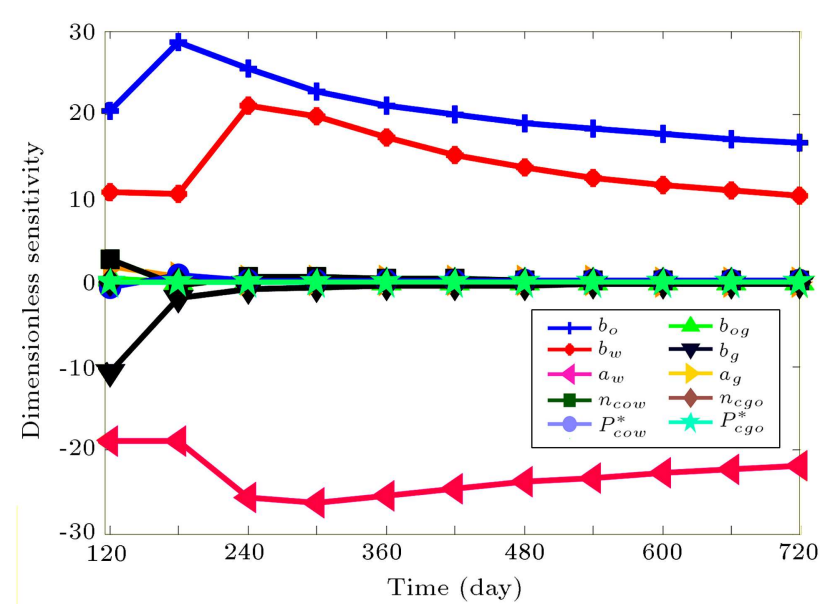

Figure 19. Dimensionless sensitivity of well I1 bottom-hole pressure to relative permeability and capillary pressure parameters.

$$
\lambda_{t}=\frac{k_{r o}}{\mu_{o}}+\frac{k_{r g}}{\mu_{g}}+\frac{k_{r w}}{\mu_{w}} .
$$

Grid blocks surrounding the injection well are almost saturated with water and oil. Therefore, the bottomhole pressure is expected to have high sensitivity to the oil-water relative permeability parameters, as it is depicted in Figure 19. Around the injection well, there is a high flow rate in the reservoir, and so the bottom-hole pressure of the injection well is insensitive to $P_{\text {cow }}^{*}$ and $n_{\text {cow }}$. As a result, a small relative error is obtained for estimation of parameters $b_{o}, b_{w}, a_{w}, n_{\text {cow }}$, and $b_{g}$ with low variances at the end of assimilation, as depicted in Figures 13 and 14 and Table 4.

The dimensionless sensitivity of the production rates of well P1 and bottom-hole pressure of well I1 to the other parameters, including $P_{\text {cow }}^{*}, b_{o g}, a_{g}, P_{c g o}^{*}$, and $n_{\text {cgo }}$, are all small. However, the information contents of these sets of data are different. Therefore, history matching of all the production rates and bottom-hole 
pressure yields an improved estimation of these parameters whose maximum relative error is $8.89 \%$, as listed in Table 4. However, as discussed above, parameters with high values of dimensionless sensitivity usually result in better estimation of the model parameters and lower uncertainties. Table 4 shows that uncertainty of the parameters $P_{c o w}^{*}, b_{o g}, a_{g}, P_{c g o}^{*}$, and $n_{c g o}$ at the end of assimilation is much greater than parameters with high dimensionless sensitivity.

\section{Conclusions}

In this study, three-phase relative permeabilities and capillary pressures were simultaneously estimated from historical data using the EnKF. The proposed technique was validated by a $2 \mathrm{D}$ three-phase heterogeneous reservoir with two different scenarios. Power-law models were used for both relative permeability and capillary pressure curves and the associated unknown parameters were adjusted sequentially toward their reference values during the assimilation process. All the unknown model parameters were accurately estimated except for the gas-oil entry capillary pressure. This is explained by calculation of the dimensionless sensitivities, which represent the sensitivity of the observation data to the unknown model parameters. It was verified that higher values of dimensionless sensitivity resulted in better estimation of the model parameters and lower uncertainty (lower variance) at the end of assimilation. Nonetheless, this consequence could be further approved by considering more complex and larger reservoir models in the future. Moreover, the impacts of availability of the endpoints and ensemble size on the filter performance were evaluated.

The second scenario in which the endpoints were unknown seemed to be a better representative of an actual history matching problem performed to characterize a real three-phase flow reservoir through simultaneous estimation of relative permeabilities and capillary pressures from production data. However, this scenario was more complicated and challenging due to its higher degree of freedom. Hence, to avoid filter divergence, a larger number of ensemble members were required, which increased the computational burden.

\section{Nomenclature}

$\begin{array}{ll}\text { A } & \text { Ensemble perturbations or anomalies } \\ a & \text { Endpoint of relative permeability curve } \\ b & \begin{array}{l}\text { Shape factor of relative permeability } \\ \text { curve }\end{array} \\ \text { D } & \text { Measurement perturbations vector } \\ \mathbf{d} & \text { Real observations } \\ \tilde{\mathbf{d}} & \text { Predicted observations } \\ \mathbf{H} & \text { Mapping operator }\end{array}$

I

K

M

$k_{r}$

m

$\mathbf{N}$

$N$

$n_{c}$

$n_{d}$

$n_{m}$

$N_{p}$

$n_{s}$

$\mathrm{O}$

P

$P_{c}$

$P_{c}^{*}$

Q

R

$R_{s}$

RRMSE

S

s

$S_{d, m}$

$S_{g c}$

$S_{g D}$

$S_{\text {org }}$

$S_{\text {orw }}$

$S_{w c}$

$S_{w D}$

std

u

$\mathbf{w}$

WOPR Well Oil Production Rate (STB/D)

WWPR Well Water Production Rate (STB/D)

WGPR Well Gas Production Rate (MSCF/D)

WBHP Well Bottom-Hole Pressure (psia)

$\mathbf{x} \quad$ State vector (augmented)

\section{Greek letters}

$\lambda_{t}$

$\mu$

Identity matrix

gain matrix

Relative permeability

Model parameters

distribution

Shape factor of capillary pressure curve

Number of observations at each assimilation cycle

Number of model parameters

Number of model states

Null matrix

Error covariance matrix

Endpoint of capillary pressure curve (psia)

Model error covariance matrix

Measurement noise covariance matrix

Relative Root-Mean-Square Error

Saturation

observation $d$ to model parameter $m$

Critical gas saturation

Dimensionless gas saturation system system

Critical water saturation

mater saturation

ard deviation

Control inputs

Model error

Measurement perturbation

Total mobility $\left(\mathrm{cp}^{-1}\right)$

Viscosity (cp) 
$\sigma_{d}$

Standard deviation of measurement error

$\sigma_{m}$ Standard deviation of model parameter

\section{Subscripts}

$\begin{array}{ll}g & \text { Gas phase } \\ g o & \text { Gas-oil system } \\ o & \text { Oil phase } \\ o w & \text { Oil-water system } \\ w & \text { Water phase }\end{array}$

\section{Superscript}

$\begin{array}{ll}a & \text { Analysis value } \\ \text { avg } & \text { Average value } \\ f & \text { Forecast value } \\ T & \text { Matrix transpose } \\ \text { true } & \text { True value }\end{array}$

\section{References}

1. Kouhanestani, S.K., Boozarjomehry, R.B. and Pishvaie, M. "Fully implicit compositional thermal simulator using rigorous multiphase calculations", Sci. Iran., 18(3), pp. 509-517 (2011). http://dx.doi.org/10.1016/j.scient.2011.05.016.

2. Jalali, J., Mohaghegh, S.D. and Gaskari, R. "Coalbed methane reservoir simulation and uncertainty analysis with artificial neural networks", Sci. Iran., 17(1), p. 65 (2010).

3. Ghaedi, M., Masihi, M., Heinemann, Z.E. and Ghazanfari, M.H. "Application of the recovery curve method for evaluation of matrix-fracture interactions", J. Nat. Gas Sci. Eng., 22, pp. 447-458 (2015). http://dx.doi.org/10.1016/j.jngse.2014.12.029.

4. Sola, B.S., Rashidi, F. and Babadagli, T. "Temperature effects on the heavy oil/water relative permeabilities of carbonate rocks", J. Petrol. Sci. Eng., 59(1), pp. 27-42 (2007). http://dx.doi.org/10.1016/j.petrol.2007. 02.005 .

5. Jahanbakhshi, S., Pishvaie, M.R. and Boozarjomehry, R.B. "Joint estimation of absolute and relative permeabilities using ensemble-based Kalman filter", $J$. Nat. Gas Sci. Eng., 26, pp. 1232-1245 (2015). http://dx.doi.org/10.1016/j.jngse.2015.08.029.

6. Parvazdavani, M., Masihi, M. and Ghazanfari, M. "Gas-oil relative permeability at near miscible conditions: An experimental and modeling approach", Sci. Iran., 20(3), pp. 626-636 (2013). http://dx.doi.org/10.1016/j.scient. 2012.11.007.

7. Archer, J.S. and Wong, S.W. "Use of a reservoir simulator to interpret laboratory waterflood data", SPE J., 13(6), pp. 343-347 (1973). http://dx.doi.org/10.2118/3551-PA.
8. Taklimy, S.Z. and Rasaei, M. "An intelligent framework for history matching an oil field with a long production history", Energ. Source Part A, 37(17), pp. 1904-1914 (2015).

http://dx.doi.org/10.1080/15567036.2011.606456.

9. Sigmund, P.M. and McCaffery, F.G. "An improved unsteady-state procedure for determining the relativepermeability characteristics of heterogeneous porous media", SPE J., 19(1), pp. 15-28 (1979). http://dx.doi.org/10.2118/6720-PA.

10. Kerig, P.D. and Watson, A.T. "Relative-permeability estimation from displacement experiments: An error analysis", SPE Reservoir Eng., 1(2), pp. 175-182 (1986). http://dx.doi.org/10.2118/12589-PA.

11. Zhang, Y. and Yang, D. "Simultaneous estimation of relative permeability and capillary pressure for tight formations using ensemble-based history matching method", Comput. Fluids, 71, pp. 446-460 (2013). http://dx.doi.org/10.1016/j.compfluid.2012.11.013.

12. Schembre, J.M. and Kovscek, A.R. "Estimation of dynamic relative permeability and capillary pressure from countercurrent imbibition experiments", Transport Porous Med., 65(1), pp. 31-51 (2006). http://dx.doi.org/10.1007/s11242-005-6092-5.

13. Santhosh, E.C. and Sangwai, J.S. "A hybrid differential evolution algorithm approach towards assisted history matching and uncertainty quantification for reservoir models", J. Petrol. Sci. Eng., 142, pp. 2135 (2016).

http://dx.doi.org/10.1016/j.petrol.2016.01.038.

14. Abdolhosseini, H. and Khamehchi, E. "History matching using traditional and finite size ensemble Kalman filter", J. Nat. Gas Sci. Eng., 27, pp. 1748-1757 (2015). http://dx.doi.org/10.1016/j.jngse.2015.10.041.

15. Chen, C., Wang, Y., Li, G. and Reynolds, A.C. "Closed-loop reservoir management on the Brugge test case", Computat. Geosci., 14(4), pp. 691-703 (2010). http://dx.doi.org/10.1007/s10596-010-9181-7.

16. Booker, J. and Ross, T. "An evolution of uncertainty assessment and quantification", Sci. Iran., 18(3), pp. 669-676 (2011). http://dx.doi.org/10.1016/j.scient.2011.04.017.

17. Emerick, A.A., History Matching and Uncertainty Characterization Using Ensemble-Based Methods, University of Tulsa, Tulsa, the United States (2012).

18. Evensen, G., Data Assimilation: The Ensemble Kalman Filter, Springer Science \& Business Media, New York (2009).

19. Gu, Y. and Oliver, D.S. "The ensemble Kalman filter for continuous updating of reservoir simulation models", J. Energ. Resour. -ASME, 128(1), pp. 79-87 (2006). http://dx.doi.org/10.1115/1.2134735.

20. Wang, Y., Li, G. and Reynolds, A.C. "Estimation of depths of fluid contacts by history matching using iterative ensemble-Kalman smoothers", SPE J., 15(2), 
pp. 509-525 (2010). http://dx.doi.org/10.2118/119056PA.

21. Li, H. and Yang, D. "Estimation of multiple petrophysical parameters for hydrocarbon reservoirs with the ensemble-based technique", Adv. Petrol. Explor. Develop, 4(2), pp. 1-17 (2012). http://dx.doi.org/10.3968

22. Li, H., Chen, S., Yang, D. and Tontiwachwuthikul, P. "Ensemble-based relative permeability estimation using B-spline model", Transport Porous Med., 85(3), pp. 703-721 (2010). http://dx.doi.org/10.1007/s11242010-9587-7.

23. Seiler, A., Evensen, G., Skjervheim, J.A., Hove, J. and Vabo, J.G. "Advanced reservoir management workflow using an EnKF based assisted history matching method", SPE Reservoir Simulation Symposium, The Woodlands, Texas (2009). http://dx.doi.org/10.2118/118906-MS.

24. Zhang, Y., Li, H. and Yang, D. "Simultaneous estimation of relative permeability and capillary pressure using ensemble-based history matching techniques", Transport Porous Med., 94(1), pp. 259-276 (2012). http://dx.doi.org/10.1007/s11242-012-0003-3.

25. Zhang, Y. and Yang, D. "Estimation of relative permeability and capillary pressure for tight formations by assimilating field production data", Inverse Probl. Sci. Eng., 22(7), pp. 1150-1175 (2014). http://dx.doi.org/10.1080/17415977.2013.856899.

26. Li, H., Chen, S., Yang, D. and Tontiwachwuthikul, P. "Estimation of relative permeability by assisted history matching using the ensemble-Kalman-filter method", J. Can. Petrol. Technol, 51(3), pp. 205-214 (2012). http://dx.doi.org/10.2118/156027-PA.

27. Masihi, M., Javanbakht, L., Horeh, F.B. and Rasaei, M. "Experimental investigation and evaluation of three-phase relative permeability models", J. Petrol. Sci. Eng., 79(1), pp. 45-53 (2011). http://dx.doi.org/10.1016/j.petrol.2011.08.017.

28. Oliveira, L.I. and Demond, A.H. "Estimation of primary drainage three-phase relative permeability for organic liquid transport in the vadose zone", J. Contam. Hydrol., 66(3), pp. 261-285 (2003). http://dx.doi.org/10.1016/S0169-7722(03)00029-9.

29. Jahanbakhshi, S., Ghazanfari, M.H. and Masihi, M. "Non-equilibrium model of three-phase flow in porous media in presence of capillary and gravity forces", J. Hydrol., 478, pp. 119-131 (2013). http://dx.doi.org/10.1016/j.jhydrol.2012.11.051.

30. Stone, H.L. "Estimation of three-phase relative permeability and residual oil data", J. Can. Petrol. Technol., 12(4), pp. 53-61 (1973). http://dx.doi.org/10.2118/7304-06.

31. Blunt, M.J. "An empirical model for three-phase relative permeability", SPE J., 5(4), pp. 435-445 (2000). http://dx.doi.org/10.2118/67950-PA.

32. Feigl, A. "Treatment of relative permeabilities for application in hydrocarbon reservoir simulation model", Nafta, 62(7-8), pp. 233-243 (2011).
33. Holmgren, C. and Morse, R. "Effect of free gas saturation on oil recovery by water flooding", J. Petrol. Technol., 3(05), pp. 135-140 (1951). http://dx.doi.org/10.2118/951135-G.

34. Aziz, K. and Settari, A., Petroleum Reservoir Simulation, Applied Science Publishers, London (1979).

35. Lewis, J.M., Lakshmivarahan, S. and Dhall, S., Dynamic Data Assimilation: A Least Squares Approach, Cambridge University Press, New York (2006). http://dx.doi.org/10.1111/j.1751-5823.2007.00030 2.x.

36. Aanonsen, S.I., Nævdal, G., Oliver, D.S., Reynolds, A.C. and Vallès, B. "The ensemble Kalman filter in reservoir engineering-a review", SPE J., 14(3), pp. 393-412 (2009). http://dx.doi.org/10.2118/117274-PA.

37. Evensen, G. "The ensemble Kalman filter for combined state and parameter estimation", IEEE Contr. Syst. Mag., 29(3), pp. 83-104 (2009).

http://dx.doi.org/10.1109/MCS.2009.932223.

38. Sakov, P. and Oke, P.R. "Implications of the form of the ensemble transformation in the ensemble square root filters", Mon. Weather Rev., 136(3), pp. 10421053 (2008). http://dx.doi.org/10.1175/2007MWR2021.1.

39. Zhang, Y., Ensemble Methods of Data Assimilation in Porous Media Flow for Non-Gaussian Prior Probability Density, University of Bergen, Norway (2015).

40. Gu, Y. and Oliver, D.S. "An iterative ensemble Kalman filter for multiphase fluid flow data assimilation", SPE J., 12(4), pp. 438-446 (2007). http://dx.doi.org/10.2118/108438-PA.

41. Thulin, K., Li, G., Aanonsen, S. and Reynolds, A. "Estimation of initial fluid contacts by assimilation of production data with EnKF", SPE Annual Technical Conference and Exhibition, Anaheim, California (2007). http://dx.doi.org/10.2118/109975-MS.

42. Kepert, J.D. "Balance-aware covariance localisation for atmospheric and oceanic ensemble Kalman filters", Computat. Geosci., 15(2), pp. 239-250 (2011). http://dx.doi.org/10.1007/s10596-010-9188-0.

43. Remy, N., Boucher, A. and Wu, J., Applied Geostatistics with SGeMS: A User's Guide, Cambridge University Press, Cambridge (2009).

44. Delijani, E.B., Pishvaie, M.R. and Boozarjomehry, R.B. "Subsurface characterization with localized ensemble Kalman filter employing adaptive thresholding", Adv. Water Resour., 69, pp. 181-196 (2014). http://dx.doi.org/10.1016/j.advwatres.2014.04.011.

45. Reynolds, A.C., Li, R. and Oliver, D.S. "Simultaneous estimation of absolute and relative permeability by automatic history matching of three-phase flow production data", J. Can. Petrol. Technol., 43(3), pp. 3746 (2004). http://dx.doi.org/10.2118/04-03-03.

46. Zhang, F., Reynolds, A.C. and Oliver, D.S. "Evaluation of the reduction in uncertainty obtained by conditioning a 3D stochastic channel to multiwell pressure data", Math. Geol., 34(6), pp. 715-742 (2002). http://dx.doi.org/10.1023/A:1019805310025. 
47. Chapra, S.C. and Canale, R.P., Numerical Methods for Engineers, McGraw-Hill, New York (2012).

\section{Biographies}

Saman Jahanbakhshi received his BS degree in Petroleum Engineering and his MS degree in Reservoir Engineering in 2010 and 2012, respectively, from Sharif University of Technology, where he is currently a PhD candidate in Petroleum Engineering. His research interests include real-time reservoir characterization, uncertainty quantification, and flow modeling in fractured reservoirs.

Mahmoud Reza Pishvaie received MS and PhD degrees in Chemical Engineering from Sharif University of Technology in 1991 and 1999, respectively, where he is now working as Faculty Member. He has been a member of the Research Council at SPGC since 2009. His research interests include optimization, control, and simulation of processing plants.

Ramin Bozorgmehry Boozarjomehry received MS and $\mathrm{PhD}$ degrees in Chemical Engineering from Sharif University of Technology and the University of Calgary in 1990 and 1997, respectively. Since then, he has been with Chemical \& Petroleum Engineering Department at Sharif University of Technology. His research interests include process simulation, control, and optimization. 